\title{
A LOCATION-ALLOCATION MODEL FOR QUALITY-BASED BLOOD SUPPLY CHAIN UNDER IER UNCERTAINTY
}

\author{
Shiva Moslemi and Seyed Hamid Reza Pasandideh*
}

\begin{abstract}
Providing blood with high quality at the lowest cost and the shortest time is main challenge of blood supply chain management. This paper presents a new model for designing a dynamic and three level blood supply chain incorporating the quality issues. The proposed model intends to locate facilities, and to determine the best strategy for blood allocation by minimizing both cost and time and maximizing the customer satisfaction based on quality of blood delivery. In order to deal with consideration of real world, intricacies such as blood freshness, both separation and apheresis extraction methods, Cross match to Transfusion ratio $(\mathrm{C} / \mathrm{T})$ and equipment failure have been involved. Also, Interval Evidential Reasoning (IER) approach is applied to handle the uncertainty of blood product demand. Since the proposed model is NP-hard, MOPSO and NSGAII algorithms are utilized to solve it. Finally, to demonstrate the applicability of the problem some numerical examples are designed in different sizes and the most favorable algorithm is determined using TOPSIS method.
\end{abstract}

Mathematics Subject Classification. 90-08.

Received June 17, 2019. Accepted April 4, 2020.

\section{INTRODUCTION}

Blood is a highly perishable product in nature that is related to human life and its supply chain is a critical problem. Among specific characteristics of blood supply chain, the quality concern is the most significant one. Nowadays, organizations are passing from the traditional supply chain management to supply chain quality management. Supply chain quality management which leads to improve performance, focuses on value creation and meeting satisfaction of customers $[17,37]$. Best practices of supply chain quality management are detected in principles of Total Quality Management (TQM). Key principles of TQM are as follows [29]:

- Define quality based on requirements of costumers.

- Follow quality at the source.

- Emphasize objective analysis instead of subjective analysis.

- Stress prevention of defects rather than identifying them.

- Concentrate on process rather than output.

- Try to achieve zero defects.

- Focus on continuous improvement.

Keywords. Blood supply chain, quality, freshness, preventive maintenance, Evidential Reasoning, location-allocation.

Department of Industrial Engineering, Faculty of Engineering, Kharazmi University, Tehran, Iran.

* Corresponding author: shr_pasandideh@khu.ac . ir 
- Inculcate that quality is responsibility of all staff.

Despite key role of quality management in supply chain; exploring quality of blood supply chains has not been widely discussed. High quality of blood supply chain is dependent on adequate financial resources, skilled and experienced staff, high quality of blood delivery, availability of holding and laboratory equipment, appropriate holding and transportation conditions, correct patient identification and effective quality management systems. Therefore, the main objective of this study is presenting a new location-allocation model to design a multi-product and multi-period blood supply chain network in which principles of TQM inclusive preventive maintenance, quality control system, inspection, staff training and performance evaluation are contributed for the first time to satisfy customer expectations. To deal with real world considerations, uncertainty of blood demand is considered and Interval Evidential Reasoning (IER) approach is used to cope with it. The objective functions of the proposed model consist of minimizing the total cost and time spent on the blood supply chain and maximizing quality of blood delivery.

In order to solve the model in large dimensions, MOPSO and NSGAII metaheuristics algorithms are executed. The following decisions are made by solving the model:

- Number and location of blood collection centers and blood transfusion centers.

- Allocation of blood collection centers to blood transfusion centers.

- Allocation of blood transfusion centers to hospitals.

- Inventory level of facilities including blood collection centers, blood transfusion centers and hospitals at each time period.

- Quantity of blood products to be produced at blood transfusion centers.

- Quantity of blood products to be transferred from each level to the next level through vehicles in the network.

- Quantity of blood shortage at hospitals.

The rest of this paper is organized as follows:

Section 2 deals with the relevant literature, research gaps, and contributions of the paper. Section 3 explains the structure of blood supply chain in detail and proposed mathematical model. the Interval Evidential Reasoning (IER) approach is described in Section 4. In Section 5, some numerical examples are designed and the suggested algorithms are implemented. Finally, the results and concluding remarks as well as directions for further studies are summarized in Section 6.

\section{Literature REVIEW}

\subsection{Location-allocation models for blood supply chain}

Pierskalla [34] presented an overview of optimization models to allocate donors to blood transfusion centers that determine the optimal number and location of centers in each region and matches supply and demand. Nagurney et al. [32] proposed a two-objective and single period optimization model for localization of blood bank systems, including collection centers, processing facilities, storage facilities, distribution centers, and hospitals with probabilistic demand and determined optimal allocations, risk supply and waste costs through the model. The their model aims to minimize total operating costs, waste disposals costs in each route, penalty costs for warehouses and costs of lack of demand, as well as minimizing the total supply risks on different routes. Nagurney et al. [31] developed the previous model to determine the optimal flow in each route and to add costs of increasing capacity. Muriel et al. [30] suggested bi-objective integer linear programming model for donor's allocation in the blood supply chain. The objective functions consider total cost minimization and amount of donors required. Hemmelmayr et al. [20] decided on the selection of hospitals that should be covered daily by blood transfusion vehicles using integer programming models. The authors also considered the uncertainty in demand and determined the amount of required blood in each hospital per day. Kaveh and Ghobadi [22] proposed an algorithm that allocates blood transfusion centers based on $p$-median methodology and metaheuristic optimization algorithms. Da et al. [10] nominated a strategic model for determining and locating donation areas 
and blood bank. They stated that the blood supply chain should be viewed as a general system, not a sub system. Daskin and Shen [11] and Shen et al. [43] developed nonlinear integer single period location-inventory models for blood supply of hospital to determine the number and location of blood transfusion centers and the inventory level in each center. Also, they proposed heuristic methods to solve the complex models. Cetin and Sarul [6] proposed a multi-objective model for locating blood banks among hospitals and clinics $u$, with the aim of minimizing location cost and distance between blood banks and hospitals. Shahin et al. [39] introduced a single period $p$-median location-allocation model for regionalization of the Turkish Red Crescent blood service, considering hierarchical services that consist of regional blood transfusion centers, blood stations and mobile units, assuming the concentration of city demand on one place. The aim of the model is to minimize the weighted distance and the number of blood stations and maximizing the covered population. Alfonso et al. [2] provided a model for locating fixed and mobile blood collection facilities in France and also examined donor management. Arvan et al. [4] designed a supply chain network composed of donated points, laboratories, central blood banks and hospitals, with the goal of minimizing total transportation and operational costs, as well as minimizing the perishability of blood. The model focuses on locating blood donation centers and blood banks and contained most of the intricacies in blood supply chain such as transshipments between demand points, blood waste and blood decomposition in laboratory facilities.

Salehipour and Sepehri [41] dealt with blood distribution management to minimize total waiting time of hospitals and medical centers and tackled a real problem containing 24 hospitals. Şahinyazan et al. [40] examined how blood from mobile centers is collected and sent to blood banks. They considered a vehicle for each district to move from a blood bank, collect donated blood from temporary centers, and then return to the same blood bank.

For designing a blood supply chain network, Zahiri and Pishvaee [47] proposed a bi-objective MILP model that minimizes the total cost and maximum unsatisfied demand, and considers blood group compatibility and uncertainty of parameters.

\subsection{Quality in supply chain management}

There are many studies on supply chain management and total quality management; but, few researches have been addressed integration of them.

Forker et al. [16] studied relation between TQM and supply chain management by combining linear (regression) and nonlinear (DEA) analyses. The results demonstrated that promoting TQM lead to better performance of the different level of supply chain. Kannan and Tan [21] examined the linkage of Total Quality Management (TQM), supply chain management and Just in time and their impact on business performance. According to results, there is a linkage between all three items at both operational and strategic levels, and focus on quality along with understanding supply chain dynamics is the main diver of performance. In 2005, Flynn and Flynn [15] examined the supply chain improved through quality management approach. In this regard, four hypotheses were developed and tested, and eventually, they approved that there was a positive relation between two approaches. Casadesu's and de Castro [5] analyzed the effect of standardized quality management system on supply chain performance improvement. They found that it is beneficial for successful SCM. Romano and Vinelli [38] addressed how to manage quality through the supply chain perspective. Outcome revealed that supply chain can meet the consumer expectations in terms of quality by the co-management of quality practices. Chen et al. [7] proposed an analytical framework for dual-channel supply chain focusing on services and quality effort. Results showed that customer loyalty to the channel has impact on the retailer's and manufacturer's services and quality strategies. Fynesa et al. [18] extended a conceptual framework to survey effect of dimensions of supply chain relationships on quality performance. To manage quality effectively in supply chain, Lin et al. [26] recognized the factors influencing the supply chain quality management (SCQM) using data collected from Hong Kong and Taiwan. The results indicated that Quality Management (QM) practices are correlated with the supplier selection and participation strategy. Lo and Yeung [27] identified critical SCQM items and clustered them in three groups: supplier selection, supplier development and supplier integration. Their study has not covered the relationship among supplier quality, buyer quality and supply quality management. Kaynak and Hartley [23] 
in their work on extension of quality management into the supply chain, found that the coverage of supplier quality management and customer focus in the QM model confirm the importance of internal and external integration for quality performance. In 2008, a study has been conducted by Foster [17] to understand the effect of practice of quality management in supply chain management. They defined SCQM and identified Key quality management variables including supplier relations, quality practices, customer focus, leadership, safety, HR practices and business results.

\subsection{Preventive maintenance in supply chain management}

Abdul Malik et al. [28] surveyed the effect of disruption factors including equipment failure, shortage of supply and lack of buying power on supply chain of Small and Medium Enterprises (SMEs). They found that equipment failure has the strongest effect on their supply chain performance. Khosrojerdi et al. [25] described a method for robust designing of power grid supply chain structure and scheduling preventive maintenance considering power supply failures scenarios. The results demonstrate that targeted failures impact on the performance of the power grid with a higher degree than random failure. Aldaihani et al. [1] determined the optimal production lot size and preventive maintenance schedules for supply chain with one producer and multiple newsvendors assuming that production process may fail at any time of production run.

\subsection{Quality (freshness) of the perishable product}

Kendall and Lee [24] applied a goal programming model to improve perishable product inventory. The model intends to reduce shortages, wastage and excessive costs, and maintain quality (freshness) of the product. Amorim et al. [3] proposed production and distribution planning as a multi-objective model for perishable products with fixed and loose shelf-life to meet customer satisfaction in terms of both economic and freshness aspects. Ogier et al. [33] presented a mixed integer programming formulation to design a short and local fresh food supply chain network. They developed effective techniques to solve the model. Pires et al. [35] addressed production planning of perishable food covering main complexities such as value of freshness, expiry dates, age dependent demand, customers' behavior and discarding costs. Gunpinar and Centeno [19] formulated integer programming models for reducing the total cost, shortage and wastage levels of blood products involving red blood cells and platelets at hospitals. They incorporated two types of patients, blood age, uncertain demand rates and crossmatch-to-transfusion ratio in the models. Farahani et al. [14] integrated production and distribution planning for perishable food products in the form of mixed integer programming modelling approach to investigate how the quality of delivered food products can be improved through decreasing the time interval between delivery and production.

As the existing literature reveals, many studies have focused on the Location-allocation of blood supply chain. Also, some papers have taken quality management and preventive maintenance in supply chain but none of them incorporate these items in blood supply chain. Moreover, only small numbers of researches have considered the complete (three level) structure of blood supply chain.

Motivated by the research gap found in the literature, we propose a mathematical model considering various dimensions of quality and different complexities of blood supply chain. The dimensions which distinguish this study from relevant works are as follows:

- Three level structure of blood supply chain including blood collection centers, blood transfusion centers and hospitals.

- Different types of blood products (whole blood, red blood cells and platelets) with different lifetime.

- Two types of collection methods including whole blood and apheresis collection.

- Quality concepts including quality of blood delivery, preventive maintenance, blood bag inspection, quality control of blood products, staff training, performance evaluation, infection disease and cross match tests, returning the products (due to expiration dates, inappropriate transportation condition, etc.), penalty of late/early delivery of blood products at hospitals and staff safety. 
- Integrated location-allocation model along with simultaneous minimization of time and cost and maximization of quality of blood delivery.

- Most important costs associated with quality (evaluation, prevention and failure), production, holding, transportation, waste, shortage, product returning, equipment, inspections, etc.

- Most important time spent on the blood supply chain: transportation, processing, cross match release period, preventive maintenance, stop times of equipment, inspection, etc.

- Quantifying quality of blood delivery as a function of the blood freshness, rate of coverage of blood demand and utility of lead time.

- Taking into account uncertainty of the demand and using IER approach to handle the uncertainty.

- Intricacies such as age-dependent blood demand, Cross-match to Transfusion ratio $(\mathrm{C} / \mathrm{T})$, blood freshness, equipment failure and vehicles with limited number and capacity.

\section{Problem definition and mathematical Model}

\subsection{Blood supply chain}

Blood supply chain is comprised of blood collection centers, blood transfusion centers and hospitals. It begins with blood collection and ends with blood transfusion to patients. In detail, supply chain process is as follows:

Blood collection centers and blood transfusion centers are constructed at potential points. First, whole blood donors go to one of the blood collection centers voluntarily, and after various examinations, they donate blood. Then, the donated blood is sent to warehouse of blood collection center. At the warehouse, the received blood will waste if holding equipment (refrigerators, etc.) fails or holding condition is not appropriate (temperature, light, etc.). At each time period, this process is repeated for a short time at blood collection centers. Thereafter, all the remaining healthy whole blood at warehouse of blood collection centers is transferred to blood transfusion centers with limited reception capacity by allocated and capacitated vehicles in less than a few hours.

In blood transfusion centers, once the whole blood is received, initial control on blood bags in terms of hemolysis, clots, and discoloration is carried out and some of the bags are returned. The remaining healthy blood bags are sent to the warehouse 1 with limited capacity and their samples are sent to the laboratory for infection disease (HIV, hepatitis, etc.) tests. The blood bags remain in warehouse 1 until the test results are determined (end of the period). There is also a possibility of waste due to equipment failure and holding conditions in this warehouse.

After determining the test results, the percentage of healthy whole blood is sent to separation unit to be converted to the blood products (random products), a fixed percentage of them is sent to Quality Control (QC) unit and the remaining bags are sent to the inspection unit. Per blood bag sent to the separation unit, two bags of blood products including platelets and red blood cells are obtained. A percentage of the products is sent to the QC control to ensure the quality of them and remaining percentage is sent to inspection unit.

On the other hand, apheresis donors also come to blood transfusion centers and their blood samples are tested, and if they are healthy, apheresis will be done. As well as random products, percentage of blood products obtained through apheresis (apheresis products) is sent to the QC unit for quality control and remaining of them is sent to inspection unit.

In inspection unit, bags of blood products including whole blood are controlled and approved products are sent to warehouse 2. Considering the key role of blood in human health, samples sent to the QC unit will be approved if none of them are defective. In the case of sample acceptance, the products in warehouse 2 are transferred to blood distribution unit and in the case of non-acceptance, inventory of warehouse 2 is considered to be defective. the healthy products along with sent whole blood are transferred to the blood distribution section.

According to demands of hospitals based on the age of the blood products in a clear time window, the blood transfusion centers transfer the blood bags containing whole blood and random and apheresis products to the hospitals by allocated vehicles. The sent blood bags are controlled at the time of receipt, and some of them are returned. Healthy blood bags are sent to unallocated warehouse and cross-match test is carried out on samples 
of them. Considering blood waste at unallocated warehouse, blood products with positive test results are sent to allocated warehouse and remained for a certain time period called the liberalization period of cross-match and eventually transfused into the patients. Figure 1 depicts structure of the blood supply chain network.

\subsection{Description of model}

In this paper, we proposed a new model for three level blood supply chain emphasizing on TQM principles to attain high quality of blood delivery at low time and cost. Best practices in blood supply chain quality management based on TQM principles considered in the model can be clustered in four critical elements of the supply chain:

Costumers. Timely delivery of appropriate blood, as close as possible to the demand, to the end customer (patients) has remarkable effects on the supply chain. Freshness is one of the main characteristics of high quality blood products in view point of costumers specially those who are vulnerable to bacterial contamination. In the model, quality of blood delivery is defined based on blood freshness, rate of coverage of blood demand and utility of lead time.

Processes. TQM principles emphasize on source inspection, process control to identify, track and improve root cause analysis. Therefore, providing infectious diseases and cross match tests and inspection of final blood products are not enough for improving quality of blood supply chain. In this paper, continuous performance evaluation and quality control of blood products are considered in addition to the mentioned items.

Equipment. Due to key role of equipment of blood supply chain (refrigerators, freezers, agitators, etc.) in processing, holding and transportation of blood, failure of this equipment at any stage of the supply chain is very costly and leads to blood wastage. However, it is inevitable to have some failures. To minimize the occurrences of such failures, ways such as preventive maintenance (PM) which is scheduled activity should be performed.

Staff. Quality is not just the responsibility of quality inspectors. Each staff should be aware of the quality problem and trained to collaborate in improving quality.

\subsubsection{Assumptions}

- The problem is multi-period, multi-product and multi-vehicle.

- Planning horizon is short time.

- Each period is equivalent to one day and the times are in hours.

- Blood products include whole blood, platelets and red blood cells.

- Platelets and red blood cells can also be produced through apheresis.

- Unit of the blood products is blood bag.

- Age of blood products is known and changes overtime.

- Blood demand at hospitals in uncertain and IER approach is used to handle the uncertainty.

- Capacity of warehouse of blood collection centers, capacity of warehouses 1, blood distribution unit and QC unit at blood transfusion centers and capacity of unallocated warehouse of hospitals are limited.

- The number of vehicles allocated to blood collection centers and blood transfusion centers is known and capacity of them is limited.

- The number of equipment at each facility is known.

- Blood collection centers and blood transfusion centers are located by solving the model, but the location of the hospitals is fixed.

- Preventive maintenance is implemented at each level. However, failure of the equipment is inevitable.

- Failure rate of equipment has exponential distribution.

- In equipment failure conditions, if the equipment can be repaired within a short time, they will be repaired and if they fail again, new equipment will be purchased.

- In equipment failure conditions, if the equipment is not repairable, they will be purchased immediately. 


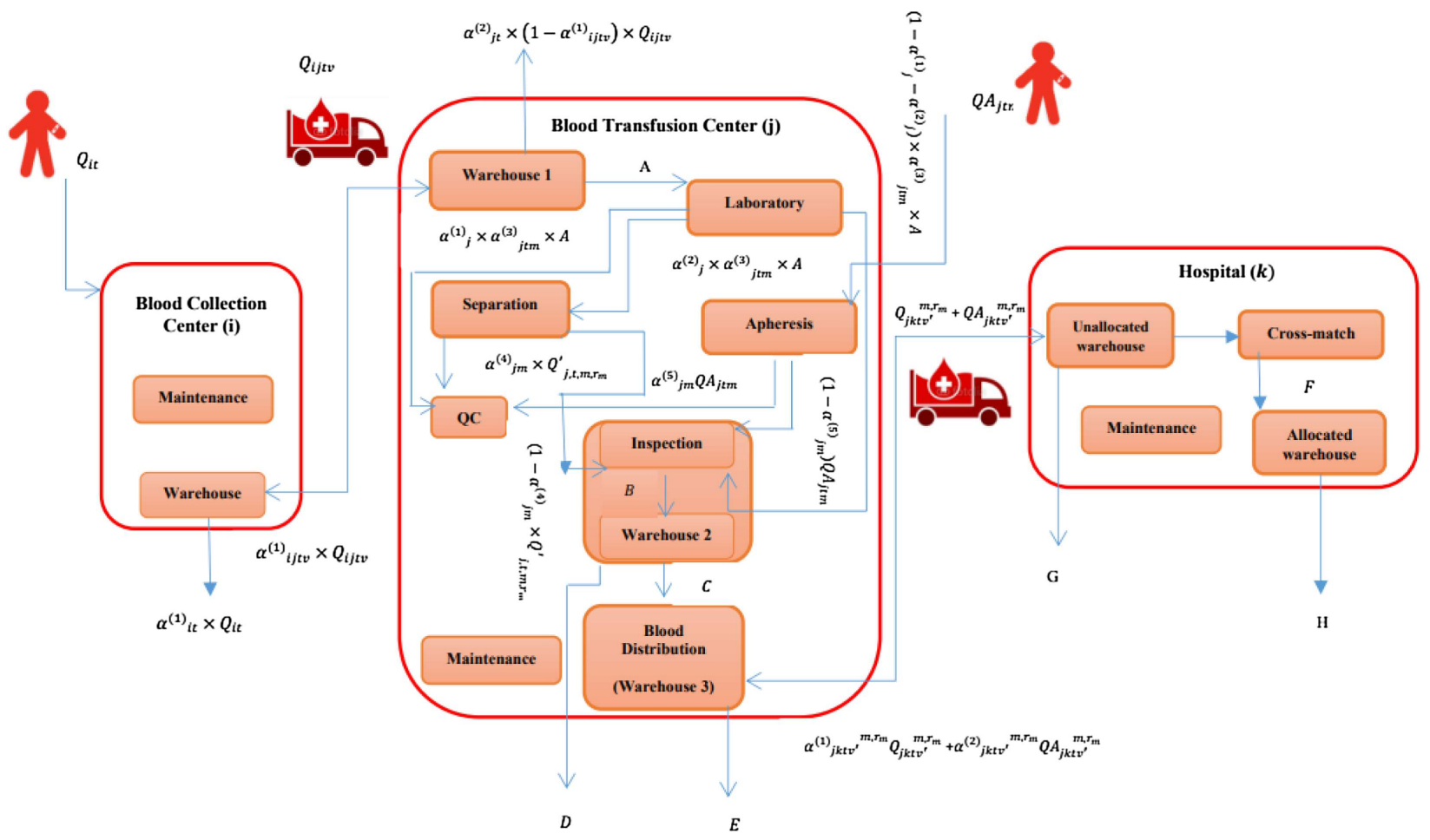

FigURE 1. Blood supply chain model. $A=\left(1-\alpha_{j t}^{(2)}\right) \times\left(1-\alpha_{i j t v}^{(1)}\right) \times Q_{i j t v}$;

$B=P_{j t m} \times\left(1-\alpha_{j m}^{(4)}\right) \times Q_{j, t, m, r_{m}}^{\prime}+P A_{j t m} \times\left(1-\alpha_{j m}^{(5)}\right) \times Q A_{j t m}+P_{j t 1} \times\left(1-\alpha_{j}^{(1)}-\alpha_{j}^{(2)}\right) \times \alpha_{j t}^{(3)} \times A ;$

$C=\left[\left(1-\alpha_{j, t, m, r_{m}}^{(2)}\right) \times P Q_{j t m} \times P_{j t m} \times\left(1-\alpha_{j m}^{(4)}\right) \times Q_{j, t, m, r_{m}}^{\prime}\right]+\left[P A_{j t m} \times\left(1-\alpha_{j m}^{(5)}\right) Q A_{j t m} \times(1-\alpha j t m) \times P Q A_{j t m}\right]+$ $\left[\left(1-\alpha_{j, t, 1, r_{1}}^{(2)}\right) \times P Q_{j t 1} \times P_{j t 1} \times\left(1-\alpha_{j}^{(1)}-\alpha_{j}^{(2)}\right) \times \alpha_{j t}^{(3)} \times A\right] ;$

$D=\left[\alpha_{j, t, m, r_{m}}^{(2)} \times P Q_{j t m}+\left(1-P Q_{j t m}\right) \frac{\left(1-E P_{j}^{(1)}\right) \times \sum_{m} \sum_{r_{m}}\left[P_{j t m} \times\left(1-\alpha_{j m}^{(4)}\right) \times Q_{j, t, m, r_{m}}^{\prime}\right]}{\sum_{m} \sum_{r_{m}} Q_{j, t, m, r_{m}}^{\prime}}\right] \times P_{j t m} \times\left(1-\alpha_{j m}^{(4)}\right) \times$

$Q_{j, t, m, r_{m}}^{\prime}+P A_{j t m} \times \alpha_{j m}^{(5)} \times Q A_{j t m} \times\left(1-\alpha_{j t m}\right) \times P Q A_{j t m}+\alpha_{j, t, 1, r_{1}}^{(2)} \times P Q_{j t 1} \times P_{j t 1} \times\left(1-\alpha_{j}^{(1)}-\alpha_{j}^{(2)}\right) \times \alpha_{f t}^{(3)} \times A+$

$\left[\alpha_{j, t, 1, r_{1}}^{(2)} P Q_{j t 1}+\left(1-P Q_{j t 1}\right) \times \sum_{j} \sum_{t} \frac{\left(1-E P_{j}^{(2)}\right) \times \sum_{i} \sum_{v} P_{j t 1} \times\left(1-\alpha_{j}^{(1)}-\alpha_{j}^{(2)}\right) \times \alpha_{j t}^{(3)} \times\left(1-\alpha_{j t}^{(2)}\right) \times\left(1-\alpha_{i j t v}^{(1)}\right) \times Q_{i j t v}}{\sum_{i} \sum_{v} \alpha_{j t}^{(3)} \times\left(1-\alpha_{j t}^{(2)}\right) \times\left(1-\alpha_{i j t v}^{(1)}\right) \times Q_{i j t v}}\right] \times$

$P_{j t 1} \times\left(1-\alpha_{j}^{(1)}-\alpha_{j}^{(2)}\right) \times \alpha_{j t}^{(3)} \times\left(1-\alpha_{j t}^{(2)}\right) \times\left(1-\alpha_{i j t v}^{(1)}\right) \times Q_{i j t v}+P A_{j t m} \times\left(1-\alpha_{j m}^{(5)}\right) Q A_{j t m} \times$

$\left[\alpha_{j t m} \times P Q A_{j t m}+\left(1-P Q A_{j t m}\right) \Sigma_{j} \Sigma_{t} \frac{\left(1-E P_{j}^{(3)}\right) \times \Sigma_{m} P A_{j t m} \times\left(1-\alpha_{j m}^{(5)}\right) Q A_{j t m}}{\sum_{m} Q A_{j t m}}\right] ;$

$E=\left[P Q_{j m} \times\left(1-\alpha_{j, t, m, r_{m}}^{(2)}\right) \times P_{j m} \times\left(1-\alpha_{j m}^{(4)}\right) \times Q_{j, t, m, r_{m}}^{\prime}+I S_{j(t-1)}^{\prime} m, r_{m}\right] \alpha_{j, t, m, r_{m}}^{(3)}+$

$\left[\sum_{i} \sum_{v} \sum_{r_{1}} P Q_{j 1} \times\left(1-\alpha_{j, t, 1, r_{1}}^{(2)}\right) \times P_{j 1} \times\left(1-\alpha_{j}^{(1)}-\alpha(2)_{j}\right) \times \alpha_{j t}^{(3)} \times A+I S_{j(t-1)}^{\prime}{ }^{1, r_{1}}\right] \alpha_{j, t, 1, r_{1}}^{(3)}+$

$\left[P Q_{j m} \times\left(1-\alpha_{j t m}\right) \times P A_{j m} \times\left(1-\alpha_{j m}^{(5)}\right) \times Q A_{j t m}+I A_{j(t-1)}{ }^{m, r_{m}}\right] \alpha_{j, t, m, r_{m}}^{(4)} ;$

$F=\left[\left(1-\alpha_{j k t v}^{m, r_{m}}\right) Q_{j k t v}^{m, r_{m}}+I n_{k(t-1)}^{m, r_{m}}\right]\left(1-\alpha_{k, t, m, r_{m}}^{(5)} P C_{k, t, m, r_{m}}+\left(1-\alpha_{j k t v^{\prime}}^{(1)}, r_{m}\right) Q_{j k t v}^{m, r_{m}}+I_{k(t-1)}^{m, r_{m}} \times\right.$

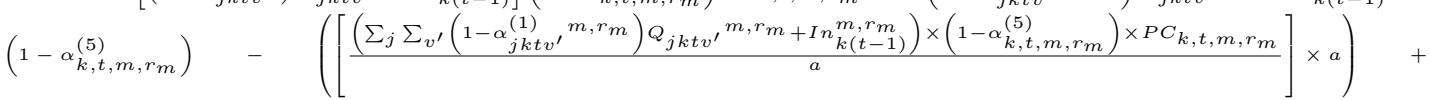
$\left[\left(1-\alpha_{j k t v^{\prime}}^{(2)} m, r_{m}\right) Q A_{j k t v}^{m, r_{m}}+I n a_{k(t-1)}^{m, r_{m}}\right]\left(1-\alpha_{k, t, m, r_{m}}^{(6)}\right) P C A_{k, t, m, r_{m}}$

$G=\left[\left(1-\alpha_{j k t v^{\prime}}^{(1)} m, r_{m}\right) Q_{j k t v^{\prime}}^{m, r_{m}}+I_{k(t-1)}^{m, r_{m}}\right] \times \alpha_{k, t, m, r_{m}}^{(5)}+\left[\left(1-\alpha_{j k t v^{\prime}}^{(2)} m^{m, r_{m}}\right) Q A_{j k t v^{\prime}}^{m, r_{m}}+I n a_{k(t-1)}^{m, r_{m}}\right] \times \alpha_{k, t, m, r_{m}}^{(6)} ;$ $H=\left(\left(1-\alpha_{j k t v^{\prime}}^{(1)}{ }^{1, r_{1}}\right) Q_{j k t v^{\prime}}^{1, r_{1}}+\operatorname{In}_{k(t-1)}^{1, r_{1}}\right) \times\left(1-\alpha_{k, t, 1, r_{1}}^{(5)}\right) \times P C_{k, t, 1, r_{1}}-Q T_{k, t, r_{1}}$. 


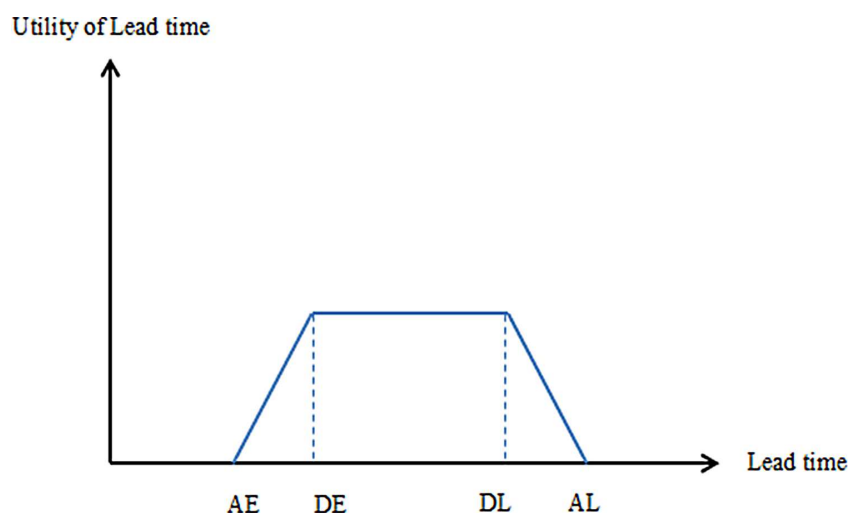

FIGURE 2. Utility of lead time in hospitals.

- At warehouses of blood collection centers and blood transfusion centers and unallocated warehouse of hospitals, blood products will be wasted if holding equipment (refrigerators, etc.) fails or holding condition is not appropriate (temperature, light, etc.)

- Holding time and cost at warehouse of blood collection centers, warehouse 2 at blood transfusion centers and allocated warehouse of hospitals are negligible.

- In each period, in blood transfusion centers, the initial tests on received whole blood is taken up to the end of the period. Therefore, in the first period, no random products are produced and only the apheresis products are prepared.

- In blood transfusion centers, due to the high cost of apheresis, the initial test is first carried out on apheresis donor blood sample and if it is healthy, the apheresis will be done. Therefore, the minimum age of the apheresis product is zero.

- Apheresis products are transferred to hospitals after approving the product in inspection and QC units and sending it to the blood distribution unit. Since all of these steps can be done in a short time, age of the sent apheresis products is at least one.

- Due to the duration of the initial test on the whole blood and the next steps, separation, inspection and quality control, age of the random products and whole blood sent to hospitals is at least two.

- In each period, whole blood, apheresis and random products are transferred to each allocated hospital simultaneously by only one vehicle.

- Utility of lead time in hospitals in terms of desirable and acceptable late and early lead time is generally illustrated in the Figure 2.

- In hospitals, whole blood transfused to patients $(\mathrm{T})$ is usually less than the prepared blood for transfusion (C).

- Blood shortage in hospital is allowed.

- Patients are adults and each adult requires an average of 8 bags of random products which is equivalent of $1 \mathrm{bag}$ of apheresis product. For this reason, the amount of random products sent to allocated warehouse of hospital is a multiple of 8 .

- In each period, each facility will provide services to at least one of its next level facility by only one vehicle.

- In each period, each facility receives at least one service.

- In each period, each vehicle is used once.

\subsubsection{Notations}

Tables 1-19 represent sets, parameters and variables used in formulation of the proposed model. 
TABLE 1. Sets of model.

\begin{tabular}{|c|c|c|c|}
\hline$I$ & Set of blood collection centers, $i=1, \ldots, I$ & $V$ & $\begin{array}{l}\text { Set of different vehicles of blood collection } \\
\text { centers, } v=1, \ldots, V\end{array}$ \\
\hline$J$ & $\begin{array}{l}\text { Set of blood transfusion centers, } j= \\
1, \ldots, J\end{array}$ & $V^{\prime}$ & $\begin{array}{l}\text { Set of different vehicles of blood transfu- } \\
\text { sion centers, } v^{\prime}=1, \ldots, V^{\prime}\end{array}$ \\
\hline$K$ & Set of hospitals, $k=1, \ldots, K$ & $O$ & $\begin{array}{l}\text { Set of different equipment of blood collec- } \\
\text { tion center, } o=1, \ldots, O\end{array}$ \\
\hline$M_{p}$ & Set of blood products, $m=1, \ldots, M_{p}$ & $P$ & $\begin{array}{l}\text { Set of different equipment of blood trans- } \\
\text { fusion center, } p=1, \ldots, P\end{array}$ \\
\hline$R_{m}$ & $\begin{array}{l}\text { Set of age of different blood products, } r_{m}= \\
0, \ldots, R_{m}\end{array}$ & $Q$ & $\begin{array}{l}\text { Set of different equipment of hospital, } q= \\
1, \ldots, Q\end{array}$ \\
\hline$T$ & Set of time periods, $t=1, \ldots, T$ & & \\
\hline
\end{tabular}

TABLE 2. General parameters.

\begin{tabular}{|c|c|c|c|}
\hline$M_{p}$ & Types of blood products & $T$ & Planning horizon time \\
\hline$n_{v}$ & $\begin{array}{l}\text { Number of vehicles of blood collection cen- } \\
\text { ters }\end{array}$ & $n_{v^{\prime}}^{\prime}$ & $\begin{array}{l}\text { Number of vehicles of blood transfusion } \\
\text { centers }\end{array}$ \\
\hline$R_{m}$ & Life time of blood product $m$ & $n e_{i o}$ & $\begin{array}{l}\text { Number of equipment } o \text { at blood collection } \\
\text { center } i\end{array}$ \\
\hline$n e_{j p}^{\prime}$ & $\begin{array}{l}\text { Number of equipment } p \text { at blood transfu- } \\
\text { sion center } j\end{array}$ & $n e_{k q}^{\prime \prime}$ & Number of equipment $q$ at hospital $k$ \\
\hline$Q_{i t}$ & $\begin{array}{l}\text { Quantity of whole blood received from } \\
\text { donors at blood collection center } i \text { and } \\
\text { period } t\end{array}$ & $Q T_{k, t, r_{1}}$ & $\begin{array}{l}\text { Quantity of whole blood with age } r_{1} \text { trans- } \\
\text { fused to patients at hospital } k \text { at period } t\end{array}$ \\
\hline
\end{tabular}

TABLE 3. Capacity parameters.

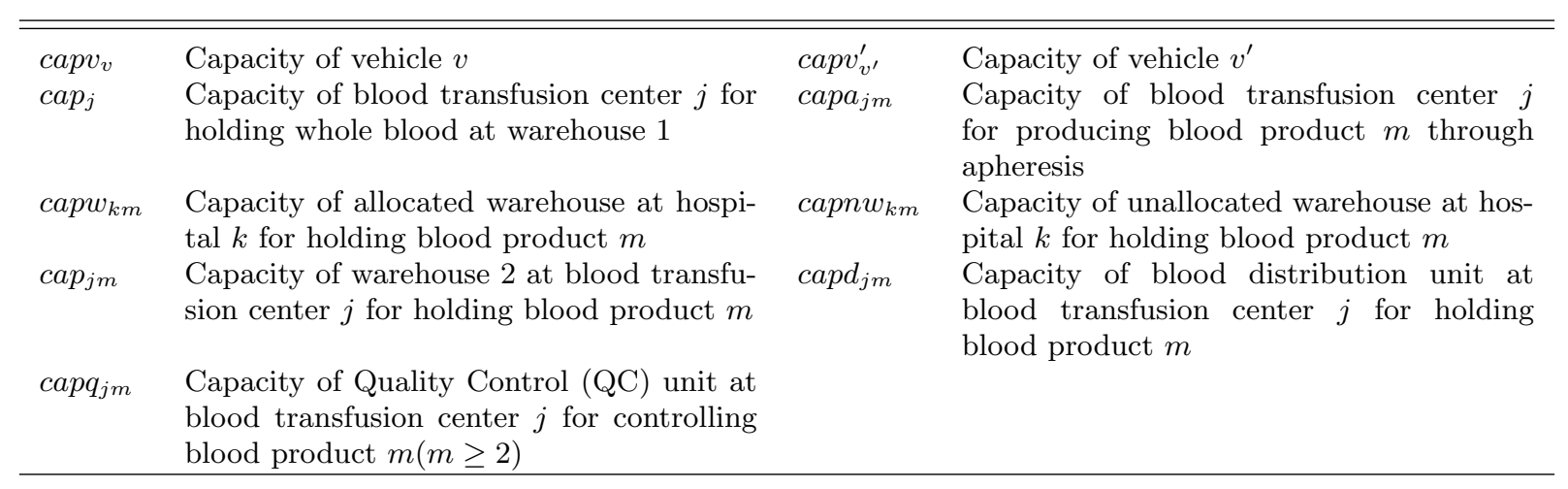

TABle 4. Demand.

\begin{tabular}{|c|c|c|c|}
\hline$d_{k, t, m, r_{m}}$ & $\begin{array}{l}\text { Demand of hospital } k \text { for blood product } \\
m \text { (including random product and whole } \\
\text { blood) with age } r_{m} \text { at period } t\end{array}$ & $d a_{k, t, m, r_{m}}$ & $\begin{array}{l}\text { Demand of hospital } k \text { for apheresis blood } \\
\text { product } m \text { with age } r_{m} \text { at period } t\end{array}$ \\
\hline
\end{tabular}

TABLE 5. Distance parameters.

\begin{tabular}{llll}
\hline \hline$d_{i j} \quad$ Distance between $i$ and $j$ (per kilometer) & $d_{j k}^{\prime}$ & Distance between $j$ and $k$ \\
\hline
\end{tabular}


TABLE 6. Cost of constructing facilities and links.

\begin{tabular}{llll}
\hline \hline$F_{i}$ & Fixed cost of opening blood collection cen- & $F_{j}$ & $\begin{array}{l}\text { Fixed cost of opening blood transfusion } \\
\text { center } j\end{array}$ \\
& ter $i$ & & \\
$L C_{i j}$ & Fixed cost of constructing a link $(i, j)$ & $L O_{i j}$ & Operating cost of constructed link on $(i, j)$ \\
$L C_{j k}^{\prime}$ & Fixed cost of constructing a link $(j, k)$ & $L O_{j k}^{\prime}$ & Operating cost of constructed link on $(j, k)$ \\
\hline
\end{tabular}

TABLE 7. Transportation cost and time.

\begin{tabular}{|c|c|c|c|}
\hline$C T_{i j}^{v}$ & $\begin{array}{l}\text { Transportation cost of one whole blood } \\
\text { unit from blood collection center } i \text { to blood } \\
\text { transfusion center } j \text { by vehicle } v \text { (per kilo- } \\
\text { meter) }\end{array}$ & $C T_{j k m}^{v^{\prime}}$ & $\begin{array}{l}\text { Transportation cost of one blood product } \\
m \text { from blood transfusion center } j \text { to hos- } \\
\text { pital } k \text { by vehicle } v^{\prime}\end{array}$ \\
\hline$t_{i j}^{v}$ & $\begin{array}{l}\text { Transportation time period between } i \text { and } \\
j \text { by vehicle } v\end{array}$ & $t_{j k}^{\prime} v^{\prime}$ & $\begin{array}{l}\text { Transportation time period between } j \text { and } \\
k \text { by vehicle } v^{\prime}\end{array}$ \\
\hline
\end{tabular}

TABle 8. Production cost and time.

\begin{tabular}{llll}
\hline \hline$C M_{j m}$ & $\begin{array}{l}\text { Production cost of one blood product } m \text { at } \\
\text { blood transfusion center } j \text { through }\end{array}$ & $C M A_{j m}$ & $\begin{array}{l}\text { Production cost of one blood product } m \text { at } \\
\text { blood transfusion center } j \text { through }\end{array}$ \\
$\begin{array}{l}\text { separation } \\
t m_{j m}\end{array}$ & $\begin{array}{l}\text { Production time period of each random } \\
\text { blood product } m \text { at blood transfusion cen- } \\
\text { ter } j\end{array}$ & $\begin{array}{l}\text { Production time period of each apheresis } \\
\text { blood product } m \text { at blood transfusion cen- } \\
\text { ter } j\end{array}$ \\
\hline
\end{tabular}

TABLE 9. Processing cost and time.

\begin{tabular}{llll}
\hline \hline$C P_{i}$ & $\begin{array}{l}\text { Processing cost at blood collection center } \\
i \text { (medical examination, ...) }\end{array}$ & $C P_{j}^{\prime}$ & $\begin{array}{l}\text { Processing cost at blood transfusion center } \\
j \text { (initial tests, ...) }\end{array}$ \\
$C P_{k}^{\prime \prime} \quad \begin{array}{l}\text { Processing cost at hospital } k \text { (categoriza- } \\
\text { tion, apheresis, } \ldots \text { ) }\end{array}$ & $\begin{array}{l}\text { Processing time period of whole blood at } \\
\text { blood collection center } i\end{array}$ \\
$t p_{j}^{\prime}$ & $\begin{array}{l}\text { Processing time period of whole blood at } \\
\text { blood transfusion center } j\end{array}$ & $t p_{k}^{\prime \prime}$ & $\begin{array}{l}\text { Processing time period of blood products } \\
\text { at hospital } k\end{array}$ \\
\hline
\end{tabular}

TABLE 10. Holding cost and time.

\begin{tabular}{llll}
\hline \hline$C H_{j m}$ & $\begin{array}{l}\text { Holding cost of one blood product } m \text { at } \\
\text { blood transfusion center } j\end{array}$ & $C H_{k m}^{\prime}$ & $\begin{array}{l}\text { Holding cost of one blood product } m \text { at } \\
\text { unallocated warehouse of hospital } k\end{array}$ \\
$t h$ & $\begin{array}{l}\text { Total holding time of whole blood at ware- } \\
\text { house } 1 \text { of blood transfusion center }\end{array}$ & $t h_{j}$ & $\begin{array}{l}\text { Holding time of blood products at blood } \\
\text { distribution unit of blood transfusion cen- } \\
\text { ter } j\end{array}$ \\
$t h$ & $\begin{array}{l}\text { Holding time of blood products at unallo- } \\
\text { cated warehouse of hospital } k \text { at period } t\end{array}$ & \\
\hline
\end{tabular}

TABLE 11. Shortage cost.

\begin{tabular}{|c|c|c|c|}
\hline$C S_{k m}$ & $\begin{array}{l}\text { Shortage cost of one random blood product } \\
m \text { at hospital } k\end{array}$ & $C S A_{k m}$ & $\begin{array}{l}\text { Shortage cost of one apheresis blood prod- } \\
\text { uct } m \text { at hospital } k\end{array}$ \\
\hline
\end{tabular}


TABle 12. Prevention cost.

\begin{tabular}{|c|c|c|c|}
\hline$C P r_{i}$ & $\begin{array}{l}\text { Fixed cost of prevention at blood collec- } \\
\text { tion center } i \text { (employee training, designing } \\
\text { quality procedures, ...) }\end{array}$ & $C P r_{j}^{\prime}$ & $\begin{array}{l}\text { Fixed cost of prevention at blood transfu- } \\
\text { sion center } j \text { (employee training, designing } \\
\text { quality procedures, ... ) }\end{array}$ \\
\hline$C P r_{k}^{\prime \prime}$ & $\begin{array}{l}\text { Fixed cost of prevention at hospital } k \\
\text { (employee training, designing quality pro- } \\
\text { cedures, ...) }\end{array}$ & & \\
\hline
\end{tabular}

TABLE 13. Cost of process evaluation.

\begin{tabular}{llll}
\hline \hline$C E V_{i}$ & $\begin{array}{l}\text { Fixed cost of process evaluation at blood } \\
\text { collection center } i\end{array}$ & $C E V_{j}^{\prime}$ & $\begin{array}{l}\text { Fixed cost of process evaluation at blood } \\
\text { transfusion center } j\end{array}$ \\
$C E V_{k}^{\prime \prime}$ & Fixed cost of process evaluation at hospital \\
$k$ & & \\
\hline
\end{tabular}

TABLE 14. Inspection parameters.

\begin{tabular}{|c|c|c|c|}
\hline$C I_{i o}$ & $\begin{array}{l}\text { Inspection cost of each equipment } O \\
\text { at blood collection center } i\end{array}$ & $C I_{j p}^{\prime}$ & $\begin{array}{l}\text { Inspection cost of equipment } p \text { at } \\
\text { blood transfusion center } j\end{array}$ \\
\hline$C I_{k q}^{\prime \prime}$ & $\begin{array}{l}\text { Inspection cost of equipment } q \text { at } \\
\text { hospital } k\end{array}$ & $C M I_{j m}$ & $\begin{array}{l}\text { Inspection cost of each blood prod- } \\
\text { uct } m \text { at inspection center of blood } \\
\text { transfusion center } j\end{array}$ \\
\hline$C Q C_{j m}$ & $\begin{array}{l}\text { Quality control cost of blood prod- } \\
\text { uct } m \text { at blood transfusion center } \\
j\end{array}$ & $C E R_{i}$ & $\begin{array}{l}\text { Fixed cost of equipment inspection } \\
\text { error at blood collection center } i\end{array}$ \\
\hline$C E R_{j}^{\prime}$ & $\begin{array}{l}\text { Fixed cost of equipment inspection } \\
\text { error at blood transfusion center } j\end{array}$ & $C E R M_{j m}$ & $\begin{array}{l}\text { Fixed cost of inspection error of } \\
\text { blood product } m \text { at blood transfu- } \\
\text { sion center } j\end{array}$ \\
\hline$C A_{j m}$ & $\begin{array}{l}\text { Acceptance cost of defective blood } \\
\text { product } m \text { including whole blood } \\
\text { and random products sent to blood } \\
\text { distribution unit at blood transfu- } \\
\text { sion center } j\end{array}$ & $C A A_{j m}$ & $\begin{array}{l}\text { Acceptance cost of defective } \\
\text { apheresis blood product } m \text { sent to } \\
\text { blood distribution unit at blood } \\
\text { transfusion center } j\end{array}$ \\
\hline$t m i_{j m}$ & $\begin{array}{l}\text { Inspection time period of each } \\
\text { blood product } m \text { at blood transfu- } \\
\text { sion center } j\end{array}$ & $t q c_{j m}$ & $\begin{array}{l}\text { Quality control time of blood prod- } \\
\text { uct } m \text { at QC unit of blood transfu- } \\
\text { sion center } j\end{array}$ \\
\hline$t c_{k m}$ & $\begin{array}{l}\text { Cross match test time for blood } \\
\text { product } m \text { at hospital } k\end{array}$ & $\alpha_{j t}^{(3)}$ & $\begin{array}{l}\text { Percentage of healthy whole blood } \\
\text { received from blood collection cen- } \\
\text { ters at laboratory of blood transfu- } \\
\text { sion center } j \text { at period } t\end{array}$ \\
\hline$\alpha_{j m}^{(4)}$ & $\begin{array}{l}\text { Percentage of random blood prod- } \\
\text { uct } m \text { transferred to QC unit of } \\
\text { blood transfusion center } j\end{array}$ & $\alpha_{j m}^{(5)}$ & $\begin{array}{l}\text { Percentage of apheresis blood prod- } \\
\text { uct } m \text { transferred to QC unit of } \\
\text { blood transfusion center } j\end{array}$ \\
\hline$\alpha_{j}^{(1)}$ & $\begin{array}{l}\text { Percentage of whole blood trans- } \\
\text { ferred to QC unit of blood trans- } \\
\text { fusion center } j\end{array}$ & $\alpha_{j}^{(2)}$ & $\begin{array}{l}\text { Percentage of whole blood devoted } \\
\text { to separation at blood transfusion } \\
\text { center } j\end{array}$ \\
\hline
\end{tabular}


TABLE 14. continued.

\begin{tabular}{|c|c|c|c|}
\hline$P_{j t m}$ & $\begin{array}{l}\text { Percentage of healthy blood prod- } \\
\text { uct } m \text { (including random product } \\
\text { and whole blood) at inspection unit } \\
\text { of blood transfusion center } j \text { at } \\
\text { period } t\end{array}$ & $P a_{j t m}$ & $\begin{array}{l}\text { Percentage of healthy apheresis } \\
\text { blood product } m \text { at inspection unit } \\
\text { of blood transfusion center } j \text { at } \\
\text { period } t\end{array}$ \\
\hline$P C_{k, t, m, r_{m}}$ & $\begin{array}{l}\text { Percentage of the crossmatch test } \\
\text { with positive result carried out on } \\
\text { blood product } m \text { (including random } \\
\text { product and whole blood) with age } \\
r_{m} \text { at hospital } k \text { at period } t\end{array}$ & $P C A_{k, t, m, r_{m}}$ & $\begin{array}{l}\text { Percentage of the crossmatch test } \\
\text { with positive result carried out on } \\
\text { apheresis blood product } m \text { with age } \\
r_{m} \text { at hospital } k \text { at period } t\end{array}$ \\
\hline$P Q A_{j t m}$ & $\begin{array}{l}\text { Acceptance probability of aphere- } \\
\text { sis blood product } m \text { at } \mathrm{QC} \text { unit of } \\
\text { blood transfusion center } j \text { at period } \\
t\end{array}$ & $P Q_{j t m}$ & $\begin{array}{l}\text { Acceptance probability of blood } \\
\text { product } m \text { (including random prod- } \\
\text { uct and whole blood) at QC unit of } \\
\text { blood transfusion center } j \text { at period } \\
t\end{array}$ \\
\hline$E P_{j}^{(1)}$ & $\begin{array}{l}\text { Average error percentage of produc- } \\
\text { tion through separation at blood } \\
\text { transfusion center } j\end{array}$ & $E P_{j}^{(2)}$ & $\begin{array}{l}\text { Average error percentage of initial } \\
\text { tests at laboratory of blood trans- } \\
\text { fusion center } j\end{array}$ \\
\hline$E P_{j}^{(3)}$ & $\begin{array}{l}\text { Average error percentage of pro- } \\
\text { duction through apheresis at blood } \\
\text { transfusion center } j\end{array}$ & & \\
\hline
\end{tabular}

TABLE 15. Waste parameters.

\begin{tabular}{|c|c|c|c|}
\hline$C W_{i}$ & $\begin{array}{l}\text { Waste cost of one whole blood unit at } \\
\text { blood collection center } i \text { (Due to space, } \\
\text { holding condition, equipment failure, etc.) }\end{array}$ & $C W_{j}^{m, r_{m}}$ & $\begin{array}{l}\text { Waste cost of one blood product } m \text { with } \\
\text { age } r_{m} \text { produced through separation at } \\
\text { blood transfusion center } j \text { (Due to separa- } \\
\text { tion, space, holding condition, equipment } \\
\text { failure, expiration date, cross match, etc.) }\end{array}$ \\
\hline$C W A_{j}^{m, r_{m}}$ & $\begin{array}{l}\text { Waste cost of one blood product } m \text { with } \\
\text { age } r_{m} \text { produced through apheresis at } \\
\text { blood transfusion center } j \text { (Due to aphere- } \\
\text { sis, space, holding condition, equipment } \\
\text { failure, expiration date, cross match, etc.) }\end{array}$ & $C W_{k}^{\prime m, r_{m}}$ & $\begin{array}{l}\text { Waste cost of one blood product } m \text { with } \\
\text { age } r_{m} \text { at hospital } k \text { (Due to canceled } \\
\text { surgery, space, holding condition, equip- } \\
\text { ment failure, high } C / T \text {, etc.) }\end{array}$ \\
\hline$\alpha_{i t}^{(1)}$ & $\begin{array}{l}\text { Waste percentage of whole blood at blood } \\
\text { collection center } i \text { at period } t\end{array}$ & $\alpha_{j t}^{(2)}$ & $\begin{array}{l}\text { Waste percentage of healthy whole blood } \\
\text { at blood transfusion center } j \text { at period } t\end{array}$ \\
\hline$\alpha_{j, t, m, r_{m}}^{(2)}$ & $\begin{array}{l}\text { Waste percentage of blood product } m \\
\text { (including random product and whole } \\
\text { blood) with age } r_{m} \text { at warehouse } 2 \text { of blood } \\
\text { transfusion center } j \text { at period } t\end{array}$ & $\alpha_{j, t, m, r_{m}}^{(3)}$ & $\begin{array}{l}\text { Waste percentage of blood product } m \\
\text { (including random product and whole } \\
\text { blood) with age } r_{m} \text { at blood distribution } \\
\text { unit of blood transfusion center } j \text { at period } \\
t\end{array}$ \\
\hline$\alpha_{j, t, m, r_{m}}^{(4)}$ & $\begin{array}{l}\text { Waste percentage of apheresis blood prod- } \\
\text { uct } m \text { with age } r_{m} \text { at blood distribution } \\
\text { unit of blood transfusion center } j \text { at period } \\
t\end{array}$ & $\alpha_{k, t, m, r_{m}}^{(5)}$ & $\begin{array}{l}\text { Waste percentage of blood product } m \\
\text { (including random product and whole } \\
\text { blood) with age } r_{m} \text { at unallocated ware- } \\
\text { house of hospital } k \text { at period } t\end{array}$ \\
\hline$\alpha_{k, t, m, r_{m}}^{(6)}$ & $\begin{array}{l}\text { Waste percentage of apheresis blood prod- } \\
\text { uct } m \text { with age } r_{m} \text { at unallocated ware- } \\
\text { house of hospital } k \text { at period } t\end{array}$ & $\alpha_{j t m}$ & $\begin{array}{l}\text { Waste percentage of apheresis blood prod- } \\
\text { uct } m \text { at warehouse } 2 \text { of blood transfusion } \\
\text { center } j \text { at period } t\end{array}$ \\
\hline
\end{tabular}


TABLE 16. Returning product parameters.

\begin{tabular}{|c|c|c|c|}
\hline$C R E P_{j k}^{m, r_{m}}$ & $\begin{array}{l}\text { Returning cost of one blood prod- } \\
\text { uct } m \text { with age } r_{m} \text { sent from blood } \\
\text { transfusion center } j \text { to hospital } k \\
\text { (Due to transportation, mismatch } \\
\text { with demand, etc.) }\end{array}$ & $C R E P_{i j}$ & $\begin{array}{l}\text { Returning cost of one whole blood } \\
\text { unit sent from blood collection cen- } \\
\text { ter } i \text { to blood transfusion center } \\
j \text { (Due to transportation, holding } \\
\text { failure, etc.) }\end{array}$ \\
\hline$\alpha_{i j t v}^{(1)}$ & $\begin{array}{l}\text { Returning percentage of whole } \\
\text { blood sent from blood collection } \\
\text { center } i \text { to blood transfusion center } \\
j \text { at period } t\end{array}$ & $\alpha_{j k t v^{\prime}}^{(1)} m, r_{m}$ & $\begin{array}{l}\text { Returning percentage of blood } \\
\text { product } m \text { (including random prod- } \\
\text { uct and whole blood) with age } r_{m} \\
\text { sent from blood transfusion center } j \\
\text { to hospital } k \text { by vehicle } v^{\prime} \text { at period } \\
t\end{array}$ \\
\hline$\alpha_{j k t v^{\prime}}^{(2)} m, r_{m}$ & $\begin{array}{l}\text { Returning percentage of apheresis } \\
\text { blood product } m \text { with age } r_{m} \text { sent } \\
\text { from blood transfusion center } j \text { to } \\
\text { hospital } k \text { by vehicle } v^{\prime} \text { at period } t\end{array}$ & & \\
\hline
\end{tabular}

TABLE 17. Lead time parameters.

\begin{tabular}{|c|c|c|c|}
\hline$Y L_{j k t v^{\prime}}$ & $\begin{array}{l}1 \text { if blood transfusion center } j \text { delivery } \\
\text { blood products to hospital } k \text { late by vehicle } \\
v^{\prime} \text { at period } t, 0 \text { otherwise }\end{array}$ & $Y E_{j k t v^{\prime}}$ & $\begin{array}{l}1 \text { if blood transfusion center } j \text { delivery } \\
\text { blood products to hospital } k \text { early by vehi- } \\
\text { cle } v^{\prime} \text { at period } t, 0 \text { otherwise }\end{array}$ \\
\hline$t d_{j k}^{m, r_{m}}$ & $\begin{array}{l}\text { Lead time of blood product } m \text { with age } r_{m} \\
\text { from blood transfusion center } j \text { to hospital } \\
k\end{array}$ & $U\left(t d a_{j k t}^{m, r_{m}}\right)$ & $\begin{array}{l}\text { Utility of Lead time of apheresis blood } \\
\text { product } \mathrm{m} \text { with age } r_{m} \text { from blood trans- } \\
\text { fusion center } j \text { to hospital } k \text { by vehicle } v \\
\text { at period } t\end{array}$ \\
\hline$t w_{i j}$ & $\begin{array}{l}\text { Lead time of whole blood from blood col- } \\
\text { lection center } i \text { to blood transfusion center } \\
j\end{array}$ & $U\left(t d_{j k t v}^{m, r_{m}}\right)$ & $\begin{array}{l}\text { Utility of Lead time of blood product } m \\
\text { (including whole blood and random prod- } \\
\text { ucts) with age } r_{m} \text { from blood transfusion } \\
\text { center } j \text { to hospital } k \text { by vehicle } v \text { at period } \\
t\end{array}$ \\
\hline$t u_{k}^{m, r_{m}}$ & $\begin{array}{l}\text { Favorable lead time of blood product } m \\
\text { with age } r_{m} \text { from blood transfusion center } \\
j \text { to hospital } k\end{array}$ & $t l_{k}^{m, r_{m}}$ & $\begin{array}{l}\text { The acceptable earliest lead time of blood } \\
\text { product } m \text { with age } r_{m} \text { from blood trans- } \\
\text { fusion center } j \text { to hospital } k\end{array}$ \\
\hline$t e_{k}^{m, r_{m}}$ & $\begin{array}{l}\text { The acceptable latest lead time (time for } \\
\text { delivery of) of blood product } m \text { with age } \\
r_{m} \text { from blood transfusion center } j \text { to hos- } \\
\text { pital } k\end{array}$ & $t d_{i o}$ & $\begin{array}{l}\text { Lead time of equipment } o \text { at blood collec- } \\
\text { tion center } i\end{array}$ \\
\hline$t d_{j p}^{\prime}$ & $\begin{array}{l}\text { Lead time of equipment } p \text { at blood trans- } \\
\text { fusion center } j\end{array}$ & $t d_{k q}^{\prime \prime}$ & Lead time of equipment $q$ at hospital $k$ \\
\hline$t e_{k t}$ & $\begin{array}{l}\text { Earliest lead time of blood products at hos- } \\
\text { pital } k \text { at period } t\end{array}$ & $C L_{k}^{m, r_{m}}$ & $\begin{array}{l}\text { Cost of acceptable late delivery of one } \\
\text { blood product } m \text { with age } r_{m} \text { at hospital } \\
k\end{array}$ \\
\hline$C E_{k}^{m, r_{m}}$ & $\begin{array}{l}\text { Cost of acceptable early delivery of one } \\
\text { blood product } m \text { with age } r_{m} \text { at hospital } \\
k\end{array}$ & & \\
\hline
\end{tabular}


TABLE 18. Preventive maintenance parameters.

\begin{tabular}{|c|c|c|c|}
\hline$C P A_{\text {io }}$ & $\begin{array}{l}\text { Cost of prevention activities for each } \\
\text { equipment } o \text { at blood collection center } i\end{array}$ & $C P A_{j p}^{\prime}$ & $\begin{array}{l}\text { Cost of prevention activities for each } \\
\text { equipment } p \text { at blood transfusion center } j\end{array}$ \\
\hline$C P A_{k q}^{\prime \prime}$ & $\begin{array}{l}\text { Cost of prevention activities for each } \\
\text { equipment } q \text { at hospital } k\end{array}$ & $C R_{i o}$ & $\begin{array}{l}\text { Repair cost of equipment } o \text { at blood collec- } \\
\text { tion center } i\end{array}$ \\
\hline$C R_{j p}^{\prime}$ & $\begin{array}{l}\text { Repair cost of equipment } p \text { at blood trans- } \\
\text { fusion center } j\end{array}$ & $C R_{k q}^{\prime \prime}$ & Repair cost of equipment $q$ at hospital $k$ \\
\hline$C B_{o}$ & Purchasing cost of each equipment $o$ & $C B_{p}^{\prime}$ & Purchasing cost of each equipment $p$ \\
\hline$C B_{q}^{\prime \prime}$ & Purchasing cost of each equipment $q$ & $t p a_{i o}$ & $\begin{array}{l}\text { Time period of preventive maintenance } \\
\text { actions for equipment } o \text { at blood collection } \\
\text { center } i\end{array}$ \\
\hline$t p a_{j p}^{\prime}$ & $\begin{array}{l}\text { Time period of preventive maintenance } \\
\text { actions for equipment } p \text { at blood transfu- } \\
\text { sion center } j\end{array}$ & $t p a_{k q}^{\prime \prime}$ & $\begin{array}{l}\text { Time period of preventive maintenance } \\
\text { actions for equipment } q \text { at hospital } k\end{array}$ \\
\hline$t m d_{i o}$ & $\begin{array}{l}\text { Stop time period of equipment } o \text { at blood } \\
\text { collection center } i \text { (including first and sec- } \\
\text { ond inspections and repairs) }\end{array}$ & $t m d_{j p}^{\prime}$ & $\begin{array}{l}\text { Stop time period of equipment } p \text { at blood } \\
\text { transfusion center } j\end{array}$ \\
\hline$t m d_{k q}^{\prime \prime}$ & $\begin{array}{l}\text { Stop time period of equipment } q \text { at hospi- } \\
\text { tal } k\end{array}$ & $\lambda_{i o}$ & $\begin{array}{l}\text { Failure rate of equipment } o \text { at blood col- } \\
\text { lection center } i \text { at time period }(0-\mathrm{T})\end{array}$ \\
\hline$\lambda_{j p}^{\prime}$ & $\begin{array}{l}\text { Failure rate of equipment } p \text { at blood trans- } \\
\text { fusion center } j \text { at time period }(0-\mathrm{T})\end{array}$ & $\lambda_{k q}^{\prime \prime}$ & $\begin{array}{l}\text { Failure rate of equipment } q \text { at hospital } k \\
\text { at time period }(0-\mathrm{T})\end{array}$ \\
\hline$N_{i o}$ & $\begin{array}{l}\text { Frequency of preventive maintenance } \\
\text { actions for equipment } o \text { at blood collection } \\
\text { center } i \text { at time period }(0-\mathrm{T})\end{array}$ & $N_{j p}^{\prime}$ & $\begin{array}{l}\text { Frequency of preventive maintenance } \\
\text { actions for equipment } p \text { at blood transfu- } \\
\text { sion center } j \text { at time period }(0-\mathrm{T})\end{array}$ \\
\hline$N_{k q}^{\prime \prime}$ & $\begin{array}{l}\text { Frequency of preventive maintenance } \\
\text { actions for equipment } q \text { at hospital } k \text { at } \\
\text { time period }(0-\mathrm{T})\end{array}$ & $P D R_{i o}$ & $\begin{array}{l}\text { Percentage of defective and repairable } \\
\text { equipment } o \text { at blood collection center } i\end{array}$ \\
\hline$P D R_{j p}^{\prime}$ & $\begin{array}{l}\text { Percentage of defective and repairable } \\
\text { equipment } p \text { at blood transfusion center } j\end{array}$ & $P D R_{k q}^{\prime \prime}$ & $\begin{array}{l}\text { Percentage of defective and repairable } \\
\text { equipment } q \text { at hospital } k\end{array}$ \\
\hline$P R_{i o}$ & $\begin{array}{l}\text { Percentage of repaired non-defective equip- } \\
\text { ment } o \text { at blood collection center } i\end{array}$ & $P R_{j p}^{\prime}$ & $\begin{array}{l}\text { Percentage of repaired non-defective equip- } \\
\text { ment } p \text { at blood transfusion center } j\end{array}$ \\
\hline$P R_{k q}^{\prime \prime}$ & $\begin{array}{l}\text { Percentage of repaired non-defective equip- } \\
\text { ment } q \text { at hospital } k\end{array}$ & & \\
\hline
\end{tabular}

\subsubsection{Mathematical model}

The model is formulated as below:

$$
\operatorname{Min} Z_{1}=\text { Total Costs. }
$$

First objective is associated with minimizing costs (see Appendix A). The costs include, respectively, the following:

Fixed cost of opening blood collection centers and blood transfusion centers, operating cost of constructing links, transportation cost from blood collection centers to blood transfusion centers, transportation cost from blood transfusion centers hospitals, preparation cost of blood products through separation and apheresis, processing cost at blood collection centers (doing medical examination including checking blood donation eligibility criteria), processing cost at blood transfusion centers including initial tests on donated blood (HIV, hepatitis, etc.), processing cost at hospitals (classification of received blood products, controlling patient's clinical and laboratory signs before and after blood transfusion, controlling patient profile before blood transfusion and matching it with blood bag), holding cost of whole blood at warehouse of blood collection centers and warehouse 1 of blood transfusion centers, holding cost of blood products at warehouse 3 of blood transfusion centers 
TABLE 19. Variables of the model.

\begin{tabular}{|c|c|c|c|}
\hline$Q_{i j t v}$ & $\begin{array}{l}\text { Quantity of whole blood sent from blood } \\
\text { collection center } i \text { to blood transfusion cen- } \\
\text { ter } j \text { at period } t\end{array}$ & $Q_{j, t, m, r_{m}}^{\prime}$ & $\begin{array}{l}\text { Quantity of produced blood product } m \\
\text { with age } r_{m} \text { through separation at blood } \\
\text { transfusion center } j \text { at period } t\end{array}$ \\
\hline$Q A_{j t m}$ & $\begin{array}{l}\text { Quantity of produced blood product } m \\
\text { through apheresis at blood transfusion cen- } \\
\text { ter } j \text { at period } t\end{array}$ & $Q \pi_{k t}^{m, r_{m}}$ & $\begin{array}{l}\text { Quantity of shortage of blood product } \\
m \text { (including random product and whole } \\
\text { blood) with age } r_{m} \text { at hospital } k \text { and } \\
\text { period } t\end{array}$ \\
\hline$Q \pi a_{k t}^{m, r_{m}}$ & $\begin{array}{l}\text { Quantity of shortage of apheresis blood } \\
\text { product } m \text { (including random product and } \\
\text { whole blood) with age } r_{m} \text { at hospital } k \text { and } \\
\text { period } t\end{array}$ & $Q_{j k t v^{\prime}}^{m, r_{m}}$ & $\begin{array}{l}\text { Quantity of blood product } m \text { (including } \\
\text { random product and whole blood) with age } \\
r_{m} \text { sent from blood transfusion center } j \text { to } \\
\text { hospital } k \text { by vehicle } v^{\prime} \text { at period } t\end{array}$ \\
\hline$Q A_{j k t v^{\prime}}^{m, r_{m}}$ & $\begin{array}{l}\text { Quantity of apheresis blood product } m \\
\text { with age } r_{m} \text { sent from blood transfusion } \\
\text { center } j \text { to hospital } k \text { by vehicle } v^{\prime} \text { at } \\
\text { period } t\end{array}$ & $Q w_{j t r_{1}}$ & $\begin{array}{l}\text { Quantity of healthy whole blood with age } \\
r_{1} \text { at laboratory of blood transfusion center } \\
j \text { at period } t\end{array}$ \\
\hline$I_{j t}$ & $\begin{array}{l}\text { Inventory level of whole blood at ware- } \\
\text { house } 1 \text { of blood transfusion center } j \text { and } \\
\text { period } t\end{array}$ & $I S_{j t}^{m, r_{m}}$ & $\begin{array}{l}\text { Inventory level of blood product } m \text { (includ- } \\
\text { ing random product and whole blood) with } \\
\text { age } r_{m} \text { at blood distribution unit of blood } \\
\text { transfusion center } j \text { at period } t\end{array}$ \\
\hline$I A_{j t}^{m, r_{m}}$ & $\begin{array}{l}\text { Inventory level of apheresis blood product } \\
m \text { with age } r_{m} \text { at blood distribution unit } \\
\text { of blood transfusion center } j \text { at period } t\end{array}$ & $I n_{k t}^{m, r_{m}}$ & $\begin{array}{l}\text { Inventory level of blood product } m \text { with } \\
\text { age } r_{m} \text { at unallocated warehouse of hospi- } \\
\text { tal } k \text { at period } t\end{array}$ \\
\hline $\operatorname{In} A_{k t}^{m, r_{m}}$ & $\begin{array}{l}\text { Inventory level of apheresis blood product } \\
m \text { with age } r_{m} \text { at unallocated warehouse } \\
\text { of hospital } k \text { at period } t\end{array}$ & $Y_{i}$ & $\begin{array}{l}1 \text { if blood collection center } i \text { is opened, } 0 \\
\text { otherwise }\end{array}$ \\
\hline$Y_{j}^{\prime}$ & $\begin{array}{l}1 \text { if blood transfusion center } j \text { is opened, } 0 \\
\text { otherwise }\end{array}$ & $Y_{i j t v}$ & $\begin{array}{l}1 \text { if link }(i, j) \text { is established through vehicle } \\
v \text { at period } t, 0 \text { otherwise }\end{array}$ \\
\hline$Y_{j k t v^{\prime}}^{\prime}$ & $\begin{array}{l}1 \text { if link }(j, k) \text { is established through vehicle } \\
v^{\prime} \text { at period } t, 0 \text { otherwise }\end{array}$ & $t h_{k t}$ & $\begin{array}{l}\text { Total holding time of blood products at } \\
\text { unallocated warehouse of hospital } k \text { at } \\
\text { period } t\end{array}$ \\
\hline
\end{tabular}

and unallocated warehouse of hospitals, shortage cost of whole blood and blood products produced through separation and apheresis at hospitals, cost of acceptable late and early delivery of blood products at hospitals, fixed costs of prevention (employee training, designing quality procedures, etc.), cost of process evaluation and equipment inspection error in each facility, fixed cost of inspection error of blood products at blood transfusion centers, waste cost of whole blood at warehouse of blood collection centers and warehouse 1 of blood transfusion centers, waste cost of random and apheresis blood products and whole blood at warehouse 2 of blood transfusion centers in both conditions of acceptance and rejection of samples at QC unit (considering error percentage in separation, apheresis and initial tests), waste cost of blood products including random, whole blood and apheresis at warehouse 3 of blood transfusion centers and unallocated warehouse of hospitals, waste cost of whole blood at hospitals because of high ratio of $\mathrm{C} / \mathrm{T}$, blood returning costs, inspection cost of blood products (apheresis, random and whole blood) at inspection unit of blood transfusion centers, fixed cost of prevention activities for equipment in each facility, cost of purchasing and repairing equipment in each facility, cost of quality control on blood products at QC unit of blood transfusion centers and cost of accepting defective blood products approved in the inspection due to error percentage in apheresis, separation and initial tests. 


$$
\begin{aligned}
& \operatorname{Min} Z_{2}=\sum_{i} \sum_{j} \sum_{t} \sum_{v} t_{i j}^{v} \times Y_{i j t v}+\sum_{j} \sum_{k} \sum_{t} \sum_{v^{\prime}}{t^{\prime v^{\prime}}}_{j k} \times Y_{j k t v^{\prime}}^{\prime}+\sum_{i} \sum_{t} t p_{i} \times Q_{i t} \times Y_{i}+\sum_{i} \sum_{j} \sum_{t} \sum_{v} t p_{j}^{\prime} \\
& \times\left(1-\alpha_{j t}^{(2)}\right) \times\left(1-\alpha_{i j t v}^{(1)}\right) \times Q_{i j t v} \times Y_{j}^{\prime}+\sum_{j} \sum_{k} \sum_{t} \sum_{v^{\prime}} \sum_{m=2} \sum_{r_{m}} C P_{k}^{\prime \prime} \\
& \times\left[\left[\frac{\left(\left(1-\alpha_{j k t v^{\prime}}^{(1)}{ }^{m, r_{m}}\right) Q_{j k t v^{\prime}}^{m, r_{m}}+I n_{k(t-1)}^{m, r_{m}}\right) \times\left(1-\alpha_{k, t, m, r_{m}}^{(5)}\right) \times P C_{k, t, m, r_{m}}}{a}\right] \times a\right. \\
& \left.+\left(\left(1-\alpha_{j k t v^{\prime}}^{(2)}{ }^{m, r_{m}}\right) Q A_{j k t v^{\prime}}^{m, r_{m}}+I n a_{k(t-1)}^{m, r_{m}}\right) \times\left(1-\alpha_{k, t, m, r_{m}}^{(6)}\right) P C A_{k, t, m, r_{m}}\right]+\sum_{j} \sum_{k} \sum_{t} \sum_{v^{\prime}} \sum_{r_{1}} \\
& \times t p_{k}^{\prime \prime} \times\left(\left(1-\alpha_{j k t v^{\prime}}^{(1)^{1, r_{1}}}\right) Q_{j k t v^{\prime}}^{1, r_{1}}+I n_{k(t-1)}^{1, r_{1}}\right) \times\left(1-\alpha_{k, t, 1, r_{1}}^{(5)}\right) \times P C_{k, t, 1, r_{1}}+\sum_{j} \sum_{t} \sum_{m=2} \sum_{r_{m}} t m_{j m} \\
& \times Q_{j, t, m, r_{m}}^{\prime}+\sum_{j} \sum_{t} \sum_{m=2} t m a_{j m} \times Q A_{j t m}+\sum_{i} \sum_{j} \sum_{t} \sum_{v} t h \times \operatorname{sgn}\left[\left(1-\alpha_{j t}^{(2)}\right) \times\left(1-\alpha_{i j t v}^{(1)}\right) \times Q_{i j t v}\right] \\
& +\sum_{j} \sum_{t} \sum_{m} \sum_{r_{m}} t h_{j} \times\left(I S_{j t}^{m, r_{m}}+I A_{j t}^{m, r_{m}}\right)+\sum_{j} \sum_{k} \sum_{t} \sum_{v^{\prime}} \sum_{m} \sum_{r_{m}} t c_{k m} \times\left[\left(1-\alpha_{j k t v^{\prime}}^{(1)}{ }^{m, r_{m}}\right)\right. \\
& \left.\times Q_{j k t v^{\prime}}^{m, r_{m}}+I n_{k(t-1)}^{m, r_{m}}\right] \times\left(1-\alpha_{k, t, m, r_{m}}^{(5)}\right)+\sum_{j} \sum_{k} \sum_{t} \sum_{v^{\prime}} \sum_{m=2} \sum_{r_{m}} t c_{k m} \times\left[\left(1-\alpha_{j k t v^{\prime}}^{(2) r^{m, r_{m}}}\right)\right. \\
& \left.\times Q A_{j k t v^{\prime}}^{m, r_{m}}+I n a_{k(t-1)}^{m, r_{m}}\right] \times\left(1-\alpha_{k, t, m, r_{m}}^{(6)}\right)+\sum_{i} \sum_{o} t p a_{i o} \times N_{i o} \times n e_{i o}+\sum_{j} \sum_{p} t p a_{j p}^{\prime} \times N_{j p}^{\prime} \\
& \times n e_{j p}^{\prime}+\sum_{k} \sum_{q} t p a_{k q}^{\prime \prime} \times N_{k q}^{\prime \prime} \times n e_{k q}^{\prime \prime}+\sum_{j} \sum_{t} \sum_{m} t m i_{j m} \times\left(Q_{j t m}^{\prime}+Q A_{j t m}\right)+\sum_{i} \sum_{o} \sum_{t} t m d_{i o} \\
& \times \lambda_{i o} \times T \times P D R_{i o}+\sum_{j} \sum_{p} \sum_{t} t m d_{j p}^{\prime} \times \lambda_{j p}^{\prime} \times T \times P D R_{j p}^{\prime}+\sum_{k} \sum_{q} \sum_{t} t m d_{k q}^{\prime \prime} \times \lambda_{k q}^{\prime \prime} \times T \times P D R_{k q}^{\prime \prime} \\
& +\sum_{i} \sum_{o} t d_{i o} \times \lambda_{i o} \times T \times\left[\left(1-P D R_{i o}\right)+P D R_{i o} \times\left(1-P R_{i o}\right)\right]+\sum_{j} \sum_{p} t d_{j p}^{\prime} \times \lambda_{j p}^{\prime} \times T \\
& \times\left[\left(1-P D R_{j p}^{\prime}\right)+P D R_{j p}^{\prime} \times\left(1-P R_{j p}^{\prime}\right)\right]+\sum_{k} \sum_{q} t d_{k q}^{\prime \prime} \times \lambda_{k q}^{\prime \prime} \times T \times\left[\left(1-P D R_{k q}^{\prime}\right)+P D R_{k q}^{\prime \prime}\right. \\
& \left.\times\left(1-P R_{k q}^{\prime \prime}\right)\right]+\sum_{j} \sum_{t} \sum_{m=2} \sum_{r_{m}} t q c_{j m} \times\left(\alpha_{j m}^{(4)} \times Q_{j, t, m, r_{m}}^{\prime}+\alpha_{j m}^{(5)} \times Q A_{j t m}\right) \\
& +\sum_{i} \sum_{j} \sum_{t} \sum_{v} t q c_{j 1} \times \alpha_{j}^{(1)} \times \alpha_{j t}^{(3)} \times\left(1-\alpha_{j t}^{(2)}\right) \times\left(1-\alpha_{i j t v}^{(1)}\right) \times Q_{i j t v} .
\end{aligned}
$$

Second objective minimizes total time in the blood supply chain. The time includes the following items:

Transportation time between facilities by available vehicles, processing time in each facility, production time of blood products through separation and apheresis, holding time of whole blood at warehouse 1 of blood transfusion centers, holding time of blood products at warehouse 3 of blood transfusion centers, holding time of blood products at unallocated warehouse of hospitals, cross-match test time at hospitals, preventive maintenance for equipment in each facility, inspection time at blood transfusion centers, stop time and lead time of equipment in each facility and time of conducting quality control on blood products at $\mathrm{QC}$ unit of blood transfusion centers. 


$$
\begin{aligned}
\operatorname{Max} Z_{3}= & \sum_{k} \sum_{t} \sum_{j} \sum_{v^{\prime}} \sum_{m} \sum_{r_{m}}\left(\frac{1+R_{m}-r_{m}}{R_{m}}\right) \times \frac{\left(1-\alpha_{j k t v^{\prime}}^{(1) \quad m, r_{m}}\right) Q_{j k t v^{\prime}}^{m, r_{m}}}{d_{k t}^{m, r_{m}}} \\
& \times U\left(t d_{j k t}^{m, r_{m}}\right)+\sum_{k} \sum_{t} \sum_{j} \sum_{v^{\prime}} \sum_{m=2} \sum_{r_{m}}\left(\frac{1+R_{m}-r_{m}}{R_{m}}\right) \\
& \times \frac{\left(1-\alpha_{j k t v^{\prime}}^{(2) r_{m}}\right) Q A_{j k t v^{\prime}}^{m, r_{m}}}{d a_{k t}^{m, r_{m}}} \times U\left(t d a_{j k t}^{m, r_{m}}\right) .
\end{aligned}
$$

The final objective function intends to maximize the quality of blood product delivered to hospitals. S.T.

$$
\begin{aligned}
& \sum_{i} \sum_{v} Q_{i j t v} \leq c a p_{j} \quad \forall j, t \\
& Q A_{j t m} \leq \operatorname{capa}_{j m} \times Y_{j}^{\prime} \quad \forall j, t, m \\
& \sum_{r_{m}} \alpha_{j m}^{(4)} \times Q_{j, t, m, r_{m}}^{\prime}+\alpha_{j m}^{(5)} \times Q A_{j t m} \leq c a p q_{j m} \quad \forall j, t, m=2, \ldots, M \\
& \sum_{i} \sum_{v} \sum_{r_{1}} \alpha_{j}^{(1)} \times \alpha_{j t}^{(3)} \times\left(1-\alpha_{j t}^{(2)}\right) \times\left(1-\alpha_{i j t v}^{(1)}\right) \times Q_{i j t v} \leq c a p q_{i 1} \quad \forall j, t \\
& \sum_{r_{m}} P_{j t m} \times\left(1-\alpha_{j m}^{(4)}\right) \times Q_{j, t, m, r_{m}}^{\prime}+P A_{j t m} \times\left(1-\alpha_{j m}^{(5)}\right) Q A_{j t m} Q A_{j t m} \leq \operatorname{cap}_{j m} \quad \forall j, t, m=2, \ldots, M \\
& \sum_{i} \sum_{v} P_{j t m} \times\left(1-\alpha_{j}^{(1)}-\alpha_{j}^{(2)}\right) \times \alpha_{j t}^{(3)} \times\left(1-\alpha_{j t}^{(2)}\right) \times\left(1-\alpha_{i j t v}^{(1)}\right) \times Q_{i j t v} \leq c a p_{j m} \quad \forall j, t, m=1 \\
& \sum_{r_{m}}\left[\left(1-\alpha_{j, t, m, r_{m}}^{(2)}\right) \times P Q_{j t m} \times P_{j t m} \times\left(1-\alpha_{j m}^{(4)}\right) \times Q_{j, t, m, r_{m}}^{\prime}+I S_{j(t-1)}^{m, r_{m}}\right] \\
& +\left[P A_{j t m} \times\left(1-\alpha_{j m}^{(5)}\right) Q A_{j t m} \times\left(1-\alpha_{j t m}\right) \times P Q A_{j t m}+I A_{j(t-1)}^{m, r_{m}}\right] \leq \operatorname{capd}_{j m} \quad \forall j, t, m=2, \ldots, M \\
& \sum_{i} \sum_{v} \sum_{r_{1}}\left[\left(1-\alpha_{j, t, 1, r_{1}}^{(2)}\right) \times P Q_{j t 1} \times P_{j t 1} \times\left(1-\alpha_{j}^{(1)}-\alpha_{j}^{(2)}\right) \times \alpha_{j t}^{(3)} \times\left(1-\alpha_{j t}^{(2)}\right) \times\left(1-\alpha_{i j t v}^{(1)}\right) \times Q_{i j t v}\right. \\
& \left.+I S_{j(t-1)}^{1, r_{1}}\right] \leq \operatorname{capd}_{j m} \quad \forall j, t, m=1 \\
& \sum_{j} \sum_{v^{\prime}} \sum_{r_{m}}\left[Q_{j k t v^{\prime}}^{m, r_{m}}+\operatorname{In}_{k(t-1)}^{m, r_{m}}\right]+\sum_{j} \sum_{v^{\prime}} \sum_{r_{m}}\left[Q A_{j k t v^{\prime}}^{m, r_{m}}+\operatorname{Ina}_{k(t-1)}^{m, r_{m}}\right] \leq \operatorname{capnw}_{k m} \quad \forall k, t, m \\
& {\left[\frac{1}{a} \times \sum_{j} \sum_{v^{\prime}} \sum_{r_{m}}\left(1-\alpha_{j k t v^{\prime}}^{(1)^{m, r_{m}}}\right) Q_{j k t v^{\prime}}^{m, r_{m}}+I_{k(t-1)}^{m, r_{m}} \times\left(1-\alpha_{k, t, m, r_{m}}^{(5)}\right) \times P C_{k, t, m, r_{m}}\right] a+\sum_{j} \sum_{v^{\prime}} \sum_{r_{m}}} \\
& \times\left(\left(1-\alpha_{j k t v^{\prime}}^{(2)^{m, r_{m}}}\right) Q A_{j k t v^{\prime}}^{m, r_{m}}+\operatorname{Ina}_{k(t-1)}^{m, r_{m}}\right)\left(1-\alpha_{k, t, m, r_{m}}^{(6)}\right) P C A_{k, t, m, r_{m}} \leq \operatorname{capw}_{k m} \quad \forall k, t, m \geq 2 \\
& \sum_{j} \sum_{v^{\prime}} \sum_{r_{1}}\left(\left(1-\alpha_{j k t v^{\prime}}^{(1)^{1, r_{1}}}\right) Q_{j k t v^{\prime}}^{1, r_{1}}+\operatorname{In}_{k(t-1)}^{1, r_{1}}\right) \times\left(1-\alpha_{k, t, 1, r_{1}}^{(5)}\right) \times P C_{k, t, 1, r_{1}} \leq \operatorname{capw}_{k m} \quad \forall k, t, m=1 \\
& Q_{i j t v} \leq \operatorname{capv}_{v} \times Y_{i j t v} \quad \forall i, j, t, v \\
& \sum_{m} \sum_{r_{m}}\left(Q_{j k t v^{\prime}}^{m, r_{m}}+Q A_{j k t v^{\prime}}^{m, r_{m}}\right) \leq c a p v_{v^{\prime}}^{\prime} \times Y_{j k t v^{\prime}}^{\prime} \quad \forall j, k, t, v^{\prime}
\end{aligned}
$$




$$
\begin{aligned}
& \sum_{j} \sum_{v} Q_{i j t v}=\left(1-\alpha_{i t}^{(1)}\right) \times Q_{i t} \quad \forall i, t \\
& Q_{j, t+1, m, r_{m}}^{\prime}=\sum_{i} \sum_{v} \alpha_{j}^{(2)} \times \alpha_{j t}^{(3)} \times\left(1-\alpha_{j t}^{(2)}\right) \times\left(1-\alpha_{i j t v}^{(1)}\right) \times Q_{i j t v} \quad \forall j, t \geq 1, m \geq 2, r_{m}=1 \\
& Q T_{k, t, r_{1}} \leq \sum_{j} \sum_{v^{\prime}}\left[\left(\left(1-\alpha_{j k t v^{\prime}}^{(1)^{1, r_{1}}}\right) Q_{j k t v^{\prime}}^{1, r_{1}}+I n_{k(t-1)}^{1, r_{1}}\right) \times\left(1-\alpha_{k, t, 1, r_{1}}^{(5)}\right) \times P C_{k, t, 1, r_{1}}\right] \quad \forall k, t, m=1, r_{1} \\
& I_{j t}^{\prime}=\sum_{i} \sum_{v}\left(1-\alpha_{j t}^{(2)}\right) \times\left(1-\alpha_{i j t v}^{(1)}\right) \times Q_{i j t v} \quad \forall j, t \\
& I S_{j t}^{\prime m, r_{m}}=\left[P Q_{j m} \times\left(1-\alpha_{j, t, m, r_{m}}^{(2)}\right) \times P_{j m} \times\left(1-\alpha_{j m}^{(4)}\right) \times Q_{j, t, m, r_{m}}^{\prime}+I S_{j(t-1)}^{\prime}{ }^{m, r_{m}}\right]\left(1-\alpha_{j, t, m, r_{m}}^{(3)}\right) \\
& -\sum_{k} \sum_{v^{\prime}} Q_{j k t v^{\prime}}^{m, r_{m}} \quad \forall j, t \geq 2, m \geq 2, r_{m}=1 \\
& I S_{j t}^{\prime 1, r_{1}}=\left[\sum_{i} \sum_{v} \sum_{r_{1}} P Q_{j 1} \times\left(1-\alpha_{j, t, 1, r_{1}}^{(2)}\right) \times P_{j 1} \times\left(1-\alpha_{j}^{(1)}-\alpha_{j}^{(2)}\right) \times \alpha_{j t}^{(3)} \times\left(1-\alpha_{j t}^{(2)}\right)\right. \\
& \left.\times\left(1-\alpha_{i j t v}^{(1)}\right) \times Q_{i j t v}+I S_{j(t-1)}^{\prime} 1, r_{1}\right]\left(1-\alpha_{j, t, 1, r_{1}}^{(3)}\right)-\sum_{k} \sum_{v^{\prime}} Q_{j k t v^{\prime}}^{1, r_{1}} \quad \forall j, t, m=1, r_{1} \\
& I A_{j t}^{m, r_{m}}=\left[P Q_{j m} \times\left(1-\alpha_{j t m}\right) \times P A_{j m} \times\left(1-\alpha_{j m}^{(5)}\right) \times Q A_{j t m}+I A_{j(t-1)}^{m, r_{m}}\right]\left(1-\alpha_{j, t, m, r_{m}}^{(4)}\right) \\
& -\sum_{k} \sum_{v^{\prime}} Q A_{j k t v^{\prime}}^{m, r_{m}} \quad \forall j, t, m \geq 2, r_{m} \\
& I n_{k t}^{1, r_{m}}=\left[\sum_{j} \sum_{v}\left(1-\alpha_{j k t v}^{m, r_{m}}\right) Q_{j k t v}^{m, r_{m}}+I n_{k(t-1)}^{m, r_{m}}\right]\left(1-\alpha_{k, t, m, r_{m}}^{(5)}\right)\left(1-P C_{k, t, m, r_{m}}\right) \quad \forall k, t, m=1, r_{1} \\
& I n_{k t}^{m, r_{m}}=\left[\left(\sum_{j} \sum_{v^{\prime}}\left(1-\alpha_{j k t v^{\prime}}^{(1)}{ }^{m, r_{m}}\right) Q_{j k t v}^{m, r_{m}}+\operatorname{In}_{k(t-1)}^{m, r_{m}}\right) \times\left(1-\alpha_{k, t, m, r_{m}}^{(5)}\right)\right] \\
& -\left[\frac{1}{a} \times\left(\sum_{j} \sum_{v^{\prime}}\left(1-\alpha_{j k t v^{\prime}}^{(1)}{ }^{m, r_{m}}\right) Q_{j k t v^{\prime}}^{m, r_{m}}+\operatorname{In}_{k(t-1)}^{m, r_{m}}\right) \times\left(1-\alpha_{k, t, m, r_{m}}^{(5)}\right) \times P C_{k, t, m, r_{m}}\right] \\
& \times a \quad \forall k, t, m \geq 2, r_{m} \\
& I n a_{k t}^{m, r_{m}}=\left[\sum_{j} \sum_{v^{\prime}}\left(1-\alpha_{j k t v^{\prime}}^{(2)}{ }^{m, r_{m}}\right) Q A_{j k t v}^{m, r_{m}}+\operatorname{In} a_{k(t-1)}^{m, r_{m}}\right]\left(1-\alpha_{k, t, m, r_{m}}^{(6)}\right)\left(1-P C A_{k, t, m, r_{m}}\right) \\
& \forall k, t, m \geq 2, r_{m} \\
& Q \pi_{k t}^{m, r_{m}}=d_{k, t, m, r_{m}}-\left[\sum_{j} \sum_{v^{\prime}}\left(1-\alpha_{j k t v^{\prime}}^{(1)}{ }^{m, r_{m}}\right) Q_{j k t v^{\prime}}^{m, r_{m}}+I n_{k(t-1)}^{m, r_{m}}\right]\left(1-\alpha_{k, t, m, r_{m}}^{(5)}\right) \times P C_{k, t, m, r_{m}} \\
& \forall k, t, m=1, r_{1}
\end{aligned}
$$




$$
\begin{aligned}
Q \pi_{k t}^{m, r_{m}}= & -d_{k, t, m, r_{m}}-\left[\frac{1}{a} \times\left(\sum_{j} \sum_{v^{\prime}}\left(1-\alpha_{j k t v^{\prime}}^{(1)^{m, r_{m}}}\right) Q_{j k t v^{\prime}}^{m, r_{m}}+I n_{k(t-1)}^{m, r_{m}}\right) \times\left(1-\alpha_{k, t, m, r_{m}}^{(5)}\right) \times P C_{k, t, m, r_{m}}\right] \\
& \times a \quad \forall k, t, m \geq 2, r_{m} \\
Q \pi a_{k t}^{m, r_{m}}= & d a_{k, t, m, r_{m}}-\left(\left[\sum_{j} \sum_{v^{\prime}}\left(1-\alpha_{j k t v^{\prime}}^{(2) r^{m, r_{m}}}\right) Q A_{j k t v^{\prime}}^{m, r_{m}}+I n a_{k(t-1)}^{m, r_{m}}\right]\left(1-\alpha_{k, t, m, r_{m}}^{(6)}\right) \times P C A_{k, t, m, r_{m}}\right)
\end{aligned}
$$

$$
\forall k, t, m \geq 2, r_{m}
$$

$$
\begin{aligned}
t h_{k t}=[24 & -\left(t e_{k t}+\sum_{j} \sum_{v^{\prime}} \sum_{m} \sum_{r_{m}} t c_{k m} \times\left[\left(1-\alpha_{j k t v^{\prime}}^{(1)}{ }^{m, r_{m}}\right) Q_{j k t v^{\prime}}^{m, r_{m}}+I n_{k(t-1)}^{m, r_{m}}\right] \times\left(1-\alpha_{k, t, m, r_{m}}^{(5)}\right)\right. \\
& \left.\left.+\sum_{j} \sum_{v^{\prime}} \sum_{m=2} \sum_{r_{m}} t c_{k m} \times\left[\left(1-\alpha_{j k t v^{\prime}}^{(2)^{m, r_{m}}}\right) Q A_{j k t v^{\prime}}^{m, r_{m}}+I n a_{k(t-1)}^{m, r_{m}}\right] \times\left(1-\alpha_{k, t, m, r_{m}}^{(6)}\right)\right)\right] \forall k, t
\end{aligned}
$$

$\sum_{i} Y_{i} \geq 1 \quad \forall i$

$\sum_{j} Y_{j}^{\prime} \geq 1 \quad \forall j$

$Y_{i j t v} \leq M \times Y_{i} \times Y_{j}^{\prime} \quad \forall i, j, t, v$

$Y_{j k t v^{\prime}}^{\prime} \leq M \times Y_{j}^{\prime} \quad \forall j, k, t, v$

$\sum_{i} \sum_{j} \sum_{v} Y_{i j t v} \leq n_{v} \quad \forall t$

$\sum_{j} \sum_{k} \sum_{v^{\prime}} Y_{j k t v^{\prime}}^{\prime} \leq n_{v^{\prime}}^{\prime} \quad \forall t$

$\sum_{i} \sum_{j} Y_{i j t v} \leq \forall v, t$

$\sum_{j} \sum_{k} Y_{j k t v^{\prime}}^{\prime} \leq 1 \quad \forall v^{\prime}, t$

$\sum_{v} Y_{i j t v} \leq 1 \quad \forall i, j, t$

$\sum_{v^{\prime}} Y_{j k t v^{\prime}}^{\prime} \leq 1 \quad \forall j, k, t$

$\sum_{j} \sum_{v} Y_{i j t v} \geq 1 \quad \forall i, t$

$\sum_{k} \sum_{v^{\prime}} Y_{j k t v^{\prime}}^{\prime} \geq 1 \quad \forall j, t$ 


$$
\begin{aligned}
& \sum_{i} \sum_{v} Y_{i j t v} \geq 1 \quad \forall j, t \\
& \sum_{j} \sum_{v} Y_{j k t v^{\prime}}^{\prime} \geq 1 \quad \forall k, t \\
& Y L_{j k t v^{\prime}} \leq Y_{j k t v^{\prime}}^{\prime} \quad \forall j, k, t, v^{\prime} \\
& Y E_{j k t v^{\prime}} \leq Y_{j k t v^{\prime}}^{\prime} \quad \forall j, k, t, v^{\prime} \\
& \sum_{i} \sum_{v} \alpha_{j t}^{(3)} \times\left(1-\alpha_{j t}^{(2)}\right) \times\left(1-\alpha_{i j t v}^{(1)}\right) \times Q_{i j t v}=Q w_{j t r_{1}} \quad \forall j, t \\
& Q_{j k t v}, Q_{j, t, m, r_{m}}^{\prime}, Q A_{j t m}, Q_{j k t v^{\prime}}^{m, r_{m}}, Q A_{j k t v^{\prime}}^{m, r_{m}}, Q \pi_{k t}^{m, r_{m}}, Q \pi a_{k t}^{m, r_{m}} I_{j t}^{\prime}, I S_{j t}^{m, r_{m}}, I A_{j t}^{m, r_{m}}, I n_{k t}^{m, r_{m}}, I n a_{k t}^{m, r_{m}} \in Z^{+} \\
& Y_{i}, Y_{j}^{\prime}, Y_{i j t v}, Y_{j k t v^{\prime}}^{\prime} \in\{0,1\} \\
& Q_{j, t, m, r_{m}}^{\prime}=Q_{j k t v^{\prime}}^{m, r_{m}}=Q w_{j t r_{1}}=0 \quad \forall t=1 \\
& Q \pi_{k t}^{m, r_{m}}>0 \quad \forall t=1 \\
& Q_{j, t, m, r_{m}}^{\prime}=Q A_{j t m}-0 \quad \forall m=1 \\
& Q_{j, t, m, r_{m}}^{\prime}=0 \quad \forall r_{m} \geq 2 \\
& Q w_{j t r_{1}}=0 \quad \forall r_{1} \geq 2 \\
& I_{j t}^{\prime}=I S_{j t}^{m, r_{m}}=I A_{j t}^{m, r_{m}}=I n_{k t}^{m, r_{m}}=I n a_{k t}^{m, r_{m}}=I S_{j t}^{\prime}{ }^{m, r_{m}}=0 \quad \forall t=1 \\
& I S_{j t}^{m, r_{m}}=I n_{k t}^{m, r_{m}}=0 \quad \forall r_{m}=1 \\
& I A_{j t}^{m, r_{m}}=I n a_{k t}^{m, r_{m}}=0 \quad \forall m=1 .
\end{aligned}
$$

Constraint (3.1) ensures that quantity of the whole blood sent from blood collection centers to warehouse 1 of each blood transfusion center at each period is less than the capacity to receive blood in the warehouses. Constraint (3.2) shows the capacity of blood transfusion centers to do apheresis at each time period. Constraints (3.3) and (3.4) indicate the capacity of QC unit in blood transfusion centers to receive apheresis and random blood products and whole blood at each time period. Constraints (3.5) and (3.6) respect the capacity of warehouse 2 in blood transfusion centers to receive apheresis and random blood products and whole blood. Constraints (3.7) and (3.8) represent the capacity of warehouse 3 in blood transfusion centers to receive apheresis and random blood products and whole blood at each time period. Constraint (3.9) states that quantity of the blood products sent from blood transfusion centers to unallocated warehouse of each hospital is less than the capacity to receive blood in the warehouses. Constraints (3.10) and (3.11) limit the amount of received apheresis and random blood products and whole blood at each time period to capacity of allocated warehouse of hospitals. Constraint (3.12) ensures that quantity of the whole blood sent from blood collection centers to the blood centers is less than the capacity of the vehicles allocated to blood collection centers. Constraint (3.13) guarantees that quantity of the blood products sent from the blood transfusion centers to the hospitals at each period is less than the capacity of the vehicles allocated to blood transfusion centers. The total amount of whole blood sent from each blood collection center to blood transfusion centers by vehicles at each time period equals the total received blood in the blood collection center (considering blood waste in blood collection center), represented by constraint (3.14). Constraint (3.15) implies that per whole blood bag sent to separation unit, 3 blood bags including platelets, plasma and red blood cells are obtained in blood transfusion centers. Constraint (3.16) shows that whole blood transfused to patients is less than whole blood in allocated warehouse of hospital at each period. Constraint (3.17) calculates whole blood inventory at warehouse 1 of blood transfusion centers in period $t$. Constraints (3.18) to (3.20) calculate blood products inventory at warehouse 3 of blood transfusion 
centers in period $t$. Constraints (3.21) to (3.23) represent blood products inventory at unallocated warehouse of blood transfusion centers at each time period. The amount of blood product shortages in each hospital in period $t$ is determined by constraints (3.24) to (3.26). Total holding time of blood products at each hospital in period $t$ is calculated by constraint (3.27). Constraint (3.28) enforces that at least one blood collection center is opened. Constraint (3.29) ensures that at least one blood transfusion center is opened. The blood collection center can transfer the whole blood to the blood transfusion centers by vehicle $v$ in period $t$, if both the blood collection center and blood transfusion center are opened, represented by constraint (3.30). Constraint (3.31) indicates that the blood collection center can transfer the whole blood to the blood transfusion centers by vehicle $v$ in period $t$, if blood transfusion center is opened. Constraint (3.32) ensures that at each time period, the total number of vehicles used for transportation of whole blood from blood collection centers to blood transfusion centers is up to the number of available vehicles. At each time period, the total number of vehicles used for transportation of blood products from blood transfusion centers to hospitals is up to the number of available vehicles, stated by constraint (3.33). Constraints (3.34) and (3.35) enforced that each vehicle is used only for one blood transportation at each time period. Constraint (3.36) and (3.37) guarantee that at each time period, only one vehicle is used for specific blood transportation. Constraint (3.38) indicates that at each time period, each blood collection center transfer whole blood to at least one blood transfusion center through a total of allocated vehicles. Constraint (3.39) lets each blood transfusion center transfer blood products to at least one hospital through a total of allocated vehicles at each time period. Constraint (3.40) limits each blood transfusion center to receive whole blood from at least one blood collection center through available vehicles at each time period. At each time period, each hospital receives blood products from at least one blood transfusion center through available vehicles, is shown by constraint (3.41). Constraints (3.42) and (3.43) express that acceptable late/early delivery will occur if link on $(j, k)$ is constructed. Amount of healthy whole blood at laboratory of blood transfusion center at period $t$ is determined by constraint (3.44). Constraints (3.45) and (3.46) clarify types of the decision variables. At the first period, quantity of random products at blood transfusion centers, quantity of blood products (including random products and whole blood) sent from blood transfusion centers to hospitals and quantity of healthy whole blood at laboratory of blood transfusion centers are zero, represented by constraint (3.47). Constraint (3.48) states that hospitals face blood shortage at the first period. Constraint (3.49) shows that random and apheresis products don't contain whole blood. Age of random products at blood transfusion centers is less than 2, indicated by constraint (3.50). According to constraint (3.51), age of healthy whole blood at laboratory of blood transfusion centers is less than 2. Constraint (3.52) enforces Initial inventory of warehouse of blood transfusion centers and hospitals is zero. Constraint (3.53) shows that age of blood product (including random product and whole blood) at blood distribution unit of blood transfusion center and unallocated warehouse of hospital is more than 1 and finally, whole blood is not included in apheresis products at blood distribution unit of blood transfusion center and unallocated warehouse of hospital, stated by constraint (3.54).

\section{Interval Evidential Reasoning (IER) APproach}

In the presented model, a number of parameters, especially those obtained from human judgment, are inevitably uncertain. Therefore, methods that handle uncertainty should be applied to make the model more realistic and produce reliable solution. For this purpose, we employ one of the most common frameworks named Interval Evidential Reasoning (IER) approach which is extension of Evidential Reasoning (ER) approach.

The ER approach has been developed for multiple attribute decision analysis (MADA) problems based upon Dempster-Shafer (D-S) theory of evidence and decision theory $[13,42,44-46]$. It can model varied types of uncertainties like incompleteness, vagueness and ignorance. In this approach, experts express their opinions in the form of belief structure including well-organized grade and belief degree. Then, the opinions are aggregated using the D-S theory. Detailed explanation of the ER approach can be found in [44].

Since an expert may be unable to provide accurate judgment by giving a single number, using interval numbers can be a sensible option that allows the experts to freely express what they have in mind. Incorporating the 
flexibility of the interval theory into the ER algorithm is called IER approach in which the grades are assigned to an individual or interval value. To simplify the understanding of this approach, we describe its procedure through an example [8].

Suppose that there are 5 experts with equal relative weight $(0 / 2)$ to estimate a parameter in IER belief structure using a set of five grades: $G=\{1,2,3,4,5\}$. So each grade in the whole range of $G$ can be assigned to the parameter and possible grading matrix is defined as follows:

$$
\text { Possible grading matrix }=\left[\begin{array}{rrrrr}
1 & 1-2 & 1-3 & 1-4 & 1-5 \\
& 2 & 2-3 & 2-4 & 2-5 \\
& & 3 & 3-4 & 3-5 \\
& & & 4 & 4-5
\end{array}\right] .
$$

Based on the above assumptions, the experts' opinions on the parameter in IER belief structure are represented as the following possible states:

- Vacillate between single grades with different belief degrees. For example, an expert estimates the parameter by giving grade 3 with belief degree of $70 \%$ and grade 4 with the belief degree of $30 \%:\{(3,70 \%),(4,30 \%)\}$.

- Vacillate between interval grades with different belief degrees. For example, an expert gives the parameter, grade [3-4] with belief degree of $20 \%$ and grade 5 with the belief degree of $50 \%$ : $\{(3-4,20 \%),(5,50 \%)\}$.

- Unawareness about the parameter. Because grading scale is [1-5], it will be modeled as: $\{(1-5), 100 \%\}$.

The notable point is that when the total belief degrees is below $100 \%$ like statement 2, remaining belief degree represents a lack of information and can be assigned to any of the grading scale.

By calculating the expected values. For example, the belief structures of the examples can be modeled by expected values as follows:

$$
\begin{aligned}
\{(3,70 \%),(4,30 \%)\} \rightarrow 3 \times 70 \%+4 \times 30 \% & =1.6=[1.41-1.41] \\
\{(3-4,20 \%),(5,50 \%)\} \rightarrow[3-4] \times 20 \%+5 \times 50 \%+[1-5] \times 30 \% & =[3.4-4.8] \\
\{(1-5,100 \%)\} \rightarrow[1-5] \times 100 \% & =[1-5]
\end{aligned}
$$

In order to decrease estimation error, the experts' opinions can be integrated. The collective estimation of the parameter $(E(S))$ is obtained from the weighted sum of the expected values of the 5 experts as follows:

$$
E(S)=\sum_{l=1}^{5} \lambda_{l} E\left(S^{l}\right)
$$

where $\lambda_{l}$ is the weight of expert $l$ and $E\left(S^{l}\right)$ is expected value of expert $l$ about the parameter.

Finally, to solve the model with these interval parameters, the lower and upper bound of the parameters are assumed to be variables and value of them is computed through the proposed meta-heuristic algorithms.

\section{Solution METHOd AND RESUlts}

The proposed model is an integer non-linear programming model and NP-hard problem. To solve such models on real scale, heuristic and meta-heuristic methods are applied. In this study, we used NSGAII and MOPSO algorithms which are the most powerful meta-heuristic algorithms for solving multi-objective optimization problems. Sections 5.1 and 5.2 summarize the procedure of these algorithm.

\subsection{Non-dominating sorting genetic algorithm-II (NSGA-II)}

The non-dominated sorting genetic algorithm II (NSGA-II), presented by Deb et al. [12], is a class of multiobjective evolutionary algorithms in which a prompt and powerful sorting procedure is applied to find Pareto front solutions. In this algorithm, off-spring population is obtained from the original one using the typical genetic 


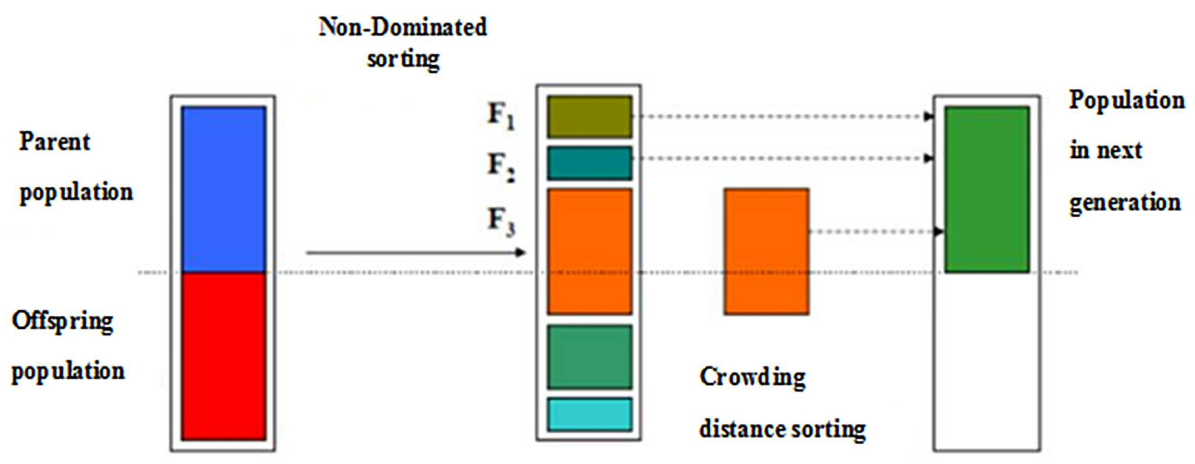

Figure 3. Mechanism of NSGA-II algorithm [36].

operators including selection, crossover, and mutation. Thereafter, the individuals are sorted based on their rank which is determined through a process called non domination sorting, and a new population is created. In the case the rank of individuals is the same, the crowding distance criterion is calculated to choose the best solutions. Figure 3 indicates mechanism of NSGA-II.

\subsection{Multi-Objective Particle Swarm Optimization (MOPSO)}

To employ the PSO algorithm for solving multi-objective optimization problems, Multi-Objective Particle Swarm Optimization (MOPSO) algorithm was proposed by Coello et al. [9]. In this algorithm, the principles of the Pareto solutions are taken into account and the population of found non-dominated solutions is kept in the repository. The repository has limited space and if the number of members exceeds its capacity, the extra ones are removed. then, each member selects a leader among the members of the repository to move on. In the next step the position of the particles is updated and the non-dominated solutions are selected to enter the repository. To enter the repository, if there is no particle inside the repository, a new particle is inserted in it, and if there is a particle in the repository while entering, and it dominates the new particle, the new particle does not enter the repository. Procedure of the MOPSO based on a dominance criterion is summarized as follows [48]:

Step 1. Initial population creation.

Step 2. Calculating objective functions.

Step 3. Separating non-dominated solutions and save them in an archive or external repository.

Step 4. Select a leader among achieve solutions for each particle.

Step 5. Update position and speed of each particle.

Step 6. Using the mutation operator.

Step 7. Updating best personal memory of each particle.

Step 8. Adding new non-dominated solutions to the archive and eliminating dominated one.

Step 9. If the number of repository members is more than the specified limit, delete extra members.

Step 10. If the algorithm is converged the operation will stop and the best particle in the swarm will be the solution of the problem. Otherwise, go back to the step 4.

\subsection{Numerical example and results}

In order to illustrate applicability of the algorithms and compare results, 15 numerical examples in different sizes (small, medium and large) are designed in which demand of hospitals in each period are under IER uncertainty. Table 20 represents the dimensions of the designed examples.

In order to set input parameters of the proposed algorithms, Taguchi method is used. According to Table 21, 3-level factors of both algorithms are determined and the experiments are designed and implemented. 
TABLE 20. Dimensions of 15 designed problems in different sizes.

\begin{tabular}{lllllllllllllll}
\hline \hline Size & Problem & $I$ & $J$ & $K$ & $O$ & $P$ & $Q$ & $V$ & $V^{\prime}$ & $T$ & $M_{p}$ & $R_{1}$ & $R_{2}$ & $R_{3}$ \\
\hline \multirow{2}{*}{ Small } & 1 & 10 & 5 & 8 & 2 & 4 & 2 & 12 & 5 & 5 & 3 & 35 & 5 & 42 \\
& 2 & 10 & 5 & 8 & 2 & 4 & 2 & 14 & 10 & 5 & 3 & 35 & 5 & 42 \\
& 3 & 10 & 5 & 8 & 2 & 4 & 2 & 16 & 5 & 5 & 3 & 35 & 5 & 42 \\
& 4 & 14 & 5 & 8 & 4 & 4 & 3 & 14 & 10 & 5 & 3 & 35 & 5 & 42 \\
& 5 & 10 & 7 & 8 & 4 & 6 & 3 & 14 & 7 & 5 & 3 & 35 & 5 & 42 \\
\hline Medium & 6 & 15 & 7 & 12 & 5 & 8 & 4 & 15 & 10 & 10 & 3 & 35 & 5 & 42 \\
& 7 & 17 & 9 & 12 & 5 & 8 & 4 & 15 & 10 & 10 & 3 & 53 & 5 & 42 \\
& 8 & 15 & 7 & 12 & 5 & 8 & 5 & 20 & 15 & 10 & 3 & 35 & 5 & 42 \\
& 9 & 17 & 7 & 12 & 5 & 10 & 5 & 25 & 20 & 10 & 3 & 35 & 5 & 42 \\
& 10 & 15 & 9 & 12 & 5 & 10 & 5 & 15 & 15 & 10 & 3 & 35 & 5 & 42 \\
\hline Large & 11 & 20 & 10 & 16 & 6 & 12 & 6 & 20 & 10 & 15 & 3 & 35 & 5 & 42 \\
& 12 & 25 & 15 & 16 & 8 & 12 & 6 & 25 & 15 & 15 & 3 & 35 & 5 & 42 \\
& 13 & 20 & 10 & 16 & 8 & 12 & 7 & 30 & 20 & 15 & 3 & 35 & 5 & 42 \\
& 14 & 25 & 10 & 16 & 10 & 14 & 7 & 30 & 10 & 15 & 3 & 35 & 5 & 42 \\
& 15 & 25 & 15 & 16 & 10 & 14 & 7 & 30 & 15 & 15 & 3 & 35 & 5 & 42 \\
\hline
\end{tabular}

TABLE 21. Different levels of parameters for proposed algorithms.

\begin{tabular}{lllll}
\hline \hline Algorithm & Factor & Level 1 & Level 2 & Level 3 \\
\hline \multirow{2}{*}{ NSGAII } & Max iteration & 100 & 150 & 200 \\
& nPop & 50 & 100 & 150 \\
& Pc & 0.7 & 0.8 & 0.9 \\
& Pm & 0.2 & 0.3 & 0.4 \\
\hline MOPSO & Max iteration & 100 & 150 & 200 \\
& nPop & 50 & 100 & 150 \\
& nRep & 50 & 80 & 100 \\
& w & 0.2 & 0.4 & 0.6 \\
& $m u$ & 0.1 & 0.15 & 0.2 \\
\hline
\end{tabular}

In the Taguchi method, Signal/Noise $(\mathrm{S} / \mathrm{N})$ ratio is applied for analysis, in which $\mathrm{S}$ refers to the response variable and $\mathrm{N}$ is standard deviation. Therefore, the higher ratio, the more favorable it is. The response variable is assumed MID, and since it is "less and better", the corresponding S/N ratio is calculated using equation (5.1).

$$
\mathrm{S} / \mathrm{N}=-10 \log \left(\frac{1}{n} \sum_{i=1}^{n} y_{i}^{2}\right) .
$$

Figures 4 and 5 depict this ratio for each algorithm.

According to the results, the parameters are adjusted. The final parameters of both algorithms are presented in Table 22.

In the next step, the problems are solved through both algorithms. Consider Figures 6 and 7, which plots solve sample of the first problem.

In order to compare the results of the algorithms, the following comparative criteria are applied:

- Number of Pareto Solution (NPS).

- Mean Ideal Distance (MID).

- Spacing (S).

- Maximum Diversity (MD).

- Time (T). 


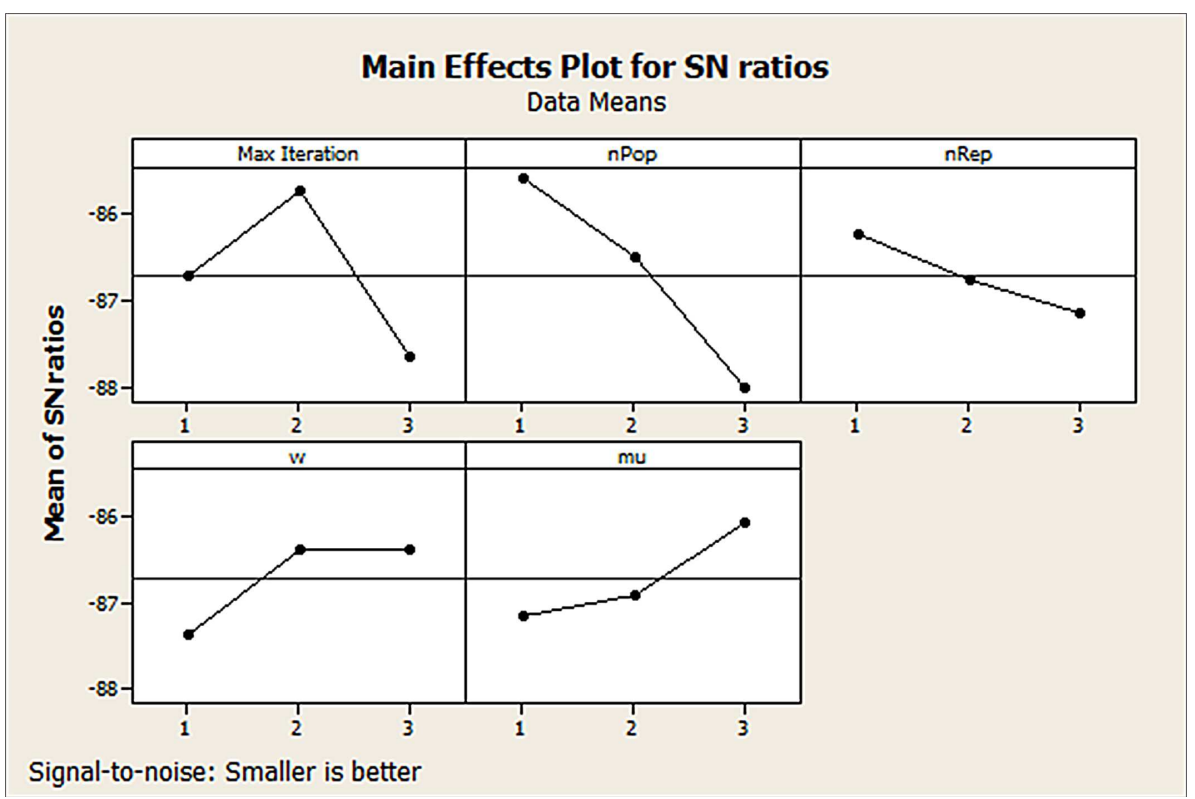

FIGURE 4. Main effects plot for SN ratios in MOPSO algorithm.

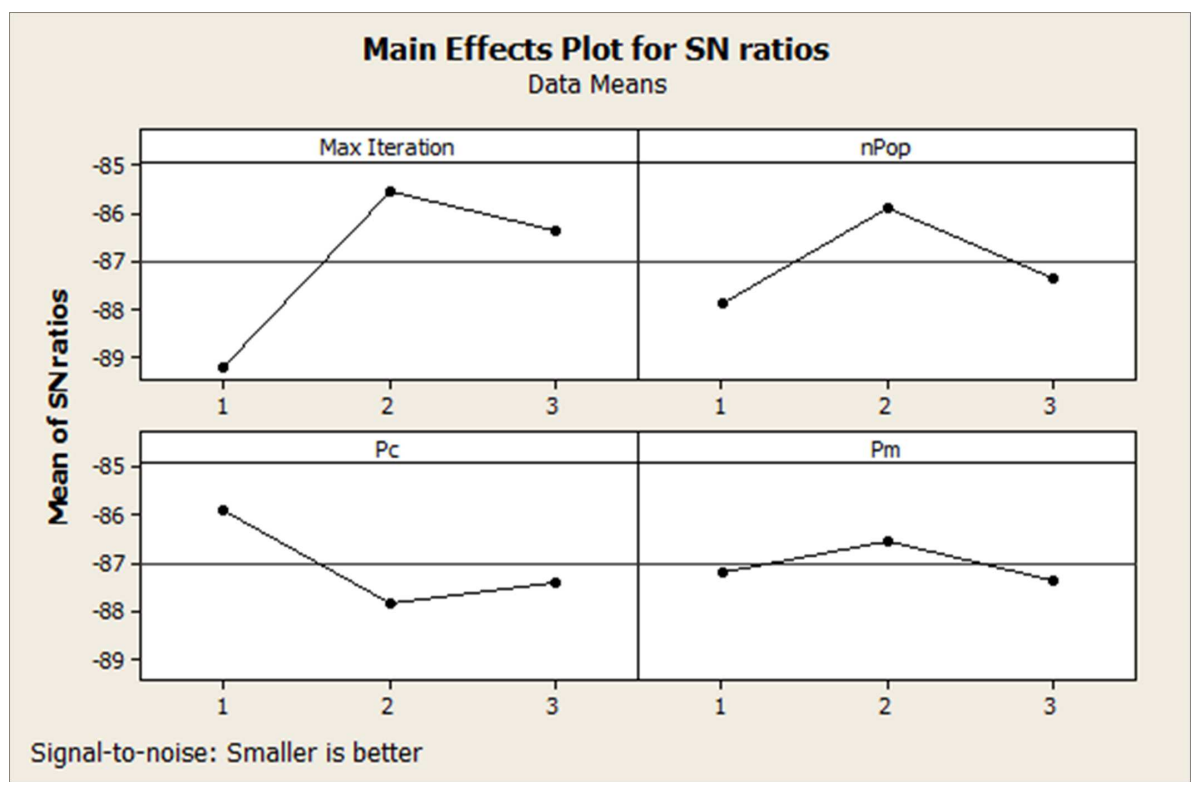

FiguRE 5. Main effects plot for SN ratios in NSGAII algorithm.

The computational results of the criteria for all designed problems can be found in Table 23 .

Figure 8 depicts average values of the criteria for both of the algorithms in different sizes.

The figure indicates that: 
TABLE 22. Final parameters of the NSGAII and MOPSO algorithms.

\begin{tabular}{lll}
\hline \hline Algorithm & Factor & Optimal level \\
\hline NSGAII & Max iteration & 150 \\
& nPop & 50 \\
& Pc & 0.7 \\
& Pm & 0.3 \\
\hline MOPSO & Max iteration & 150 \\
& nPop & 50 \\
& nRep & 50 \\
& w & 0.4 \\
& $m u$ & 0.2 \\
\hline
\end{tabular}

\section{NSGAII}

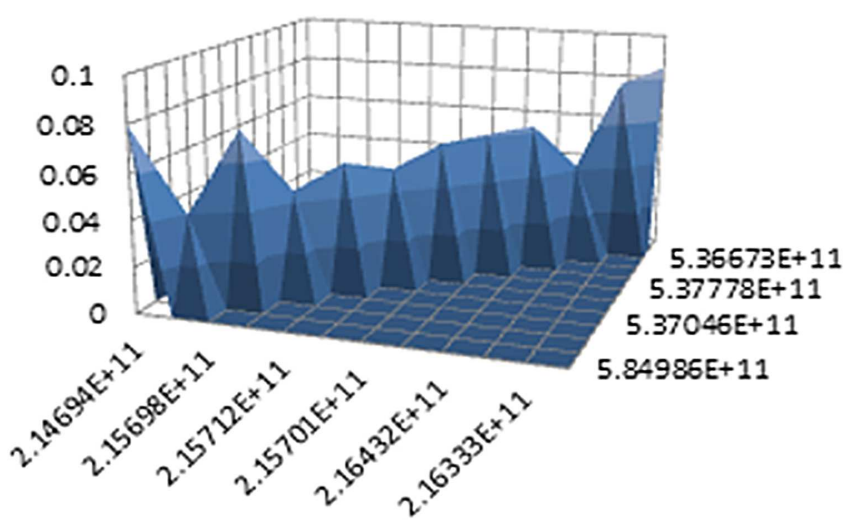

Figure 6. Pareto solutions of problem1 using NSGAII.

\section{MOPSO}

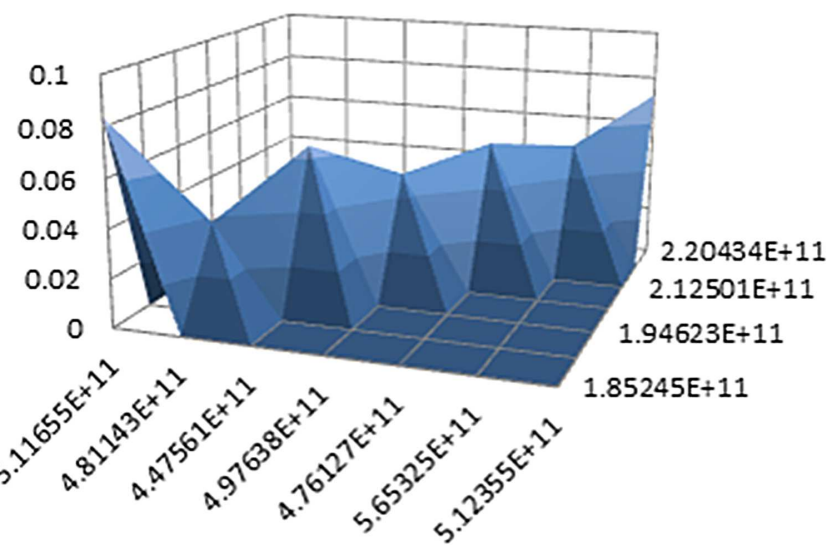

Figure 7. Pareto solutions of problem1 using MOPSO. 
TABLE 23. Value of comparative criteria in both NSGAII and MOPSO algorithms for all problems.

\begin{tabular}{|c|c|c|c|c|c|c|c|c|c|c|c|}
\hline \multirow[t]{2}{*}{ Size } & \multirow[t]{2}{*}{ Problem } & \multicolumn{2}{|c|}{ NPS } & \multicolumn{2}{|c|}{ MD } & \multicolumn{2}{|c|}{$\mathrm{S}$} & \multicolumn{2}{|c|}{ MID } & \multicolumn{2}{|c|}{$\mathrm{T}$} \\
\hline & & NSGAII & MOPSO & NSGAII & MOPSO & NSGAII & MOPSO & NSGAII & MOPSO & NSGAII & MOPSO \\
\hline \multirow[t]{5}{*}{ Small } & 1 & 12 & 7 & $9117592 / 69$ & $7748589 / 37$ & $763554 / 81$ & $411225 / 72$ & $54233279 / 99$ & $47499617 / 4$ & $316 / 37$ & $222 / 85$ \\
\hline & 2 & 13 & 28 & $14344674 / 3$ & $5375737 / 52$ & $945291 / 25$ & $727305 / 66$ & $69988160 / 55$ & $67456149 / 75$ & $369 / 53$ & $260 / 27$ \\
\hline & 3 & 18 & 25 & $6435724 / 42$ & $5109496 / 12$ & $371631 / 04$ & $295536 / 92$ & $63708438 / 42$ & $48453812 / 8$ & $330 / 4$ & $235 / 12$ \\
\hline & 4 & 4 & 8 & $27114132 / 47$ & $24429550 / 51$ & $2134940 / 37$ & $984592 / 6$ & $76268698 / 72$ & $75415370 / 69$ & $398 / 01$ & $279 / 07$ \\
\hline & 5 & 12 & 18 & $18330696 / 28$ & $16175712 / 64$ & $826713 / 52$ & $912498 / 28$ & $70240272 / 4$ & $65835325 / 39$ & $382 / 65$ & $264 / 46$ \\
\hline \multirow[t]{5}{*}{ Medium } & 6 & 16 & 22 & $10752102 / 5$ & $14273177 / 9$ & $5583377 / 2$ & $3108264 / 25$ & $75101842 / 01$ & $76978452 / 2$ & $459 / 13$ & $299 / 85$ \\
\hline & 7 & 22 & 12 & $19653599 / 35$ & $25791281 / 21$ & $5685405 / 3$ & $5188851 / 5$ & $87798877 / 23$ & $92296964 / 81$ & $487 / 77$ & $330 / 54$ \\
\hline & 8 & 28 & 26 & $26105563 / 14$ & $27177817 / 78$ & $10963117 / 65$ & $7128688 / 5$ & $131433510 / 53$ & $142207662 / 11$ & $521 / 96$ & $381 / 08$ \\
\hline & 9 & 31 & 24 & $42890930 / 07$ & $45629894 / 56$ & $11485864 / 23$ & $12818270 / 14$ & $162762841 / 9$ & $180284709 / 44$ & $565 / 55$ & $427 / 46$ \\
\hline & 10 & 19 & 12 & $36737414 / 4$ & $35653107 / 25$ & $11579064 / 11$ & $7568311 / 42$ & $112030960 / 58$ & $115279603 / 62$ & $532 / 09$ & $397 / 4$ \\
\hline \multirow[t]{5}{*}{ Large } & 11 & 50 & 41 & $16254926 / 3$ & $20054970 / 06$ & $10165010 / 44$ & $6138545 / 23$ & $191419636 / 7$ & $193440815 / 15$ & $620 / 17$ & $475 / 88$ \\
\hline & 12 & 41 & 27 & $33102550 / 85$ & $18100810 / 82$ & $14215482 / 04$ & $24185624 / 94$ & $270839725 / 41$ & $274063133 / 75$ & $654 / 26$ & $515 / 09$ \\
\hline & 13 & 24 & 25 & $17157902 / 13$ & $40993443 / 19$ & $11785693 / 9$ & $13312976 / 6$ & $240960255 / 16$ & $261716489 / 04$ & $570 / 59$ & $427 / 74$ \\
\hline & 14 & 52 & 34 & $49132499 / 66$ & $51530620 / 8$ & $11756202 / 1$ & $29333939 / 83$ & $316074921 / 14$ & $321207145 / 65$ & $619 / 84$ & $453 / 28$ \\
\hline & 15 & 41 & 30 & $65236100 / 78$ & $65500324 / 2$ & $20768835 / 37$ & $35382583 / 2$ & $480675438 / 04$ & $491819616 / 57$ & $657 / 53$ & $482 / 3$ \\
\hline
\end{tabular}

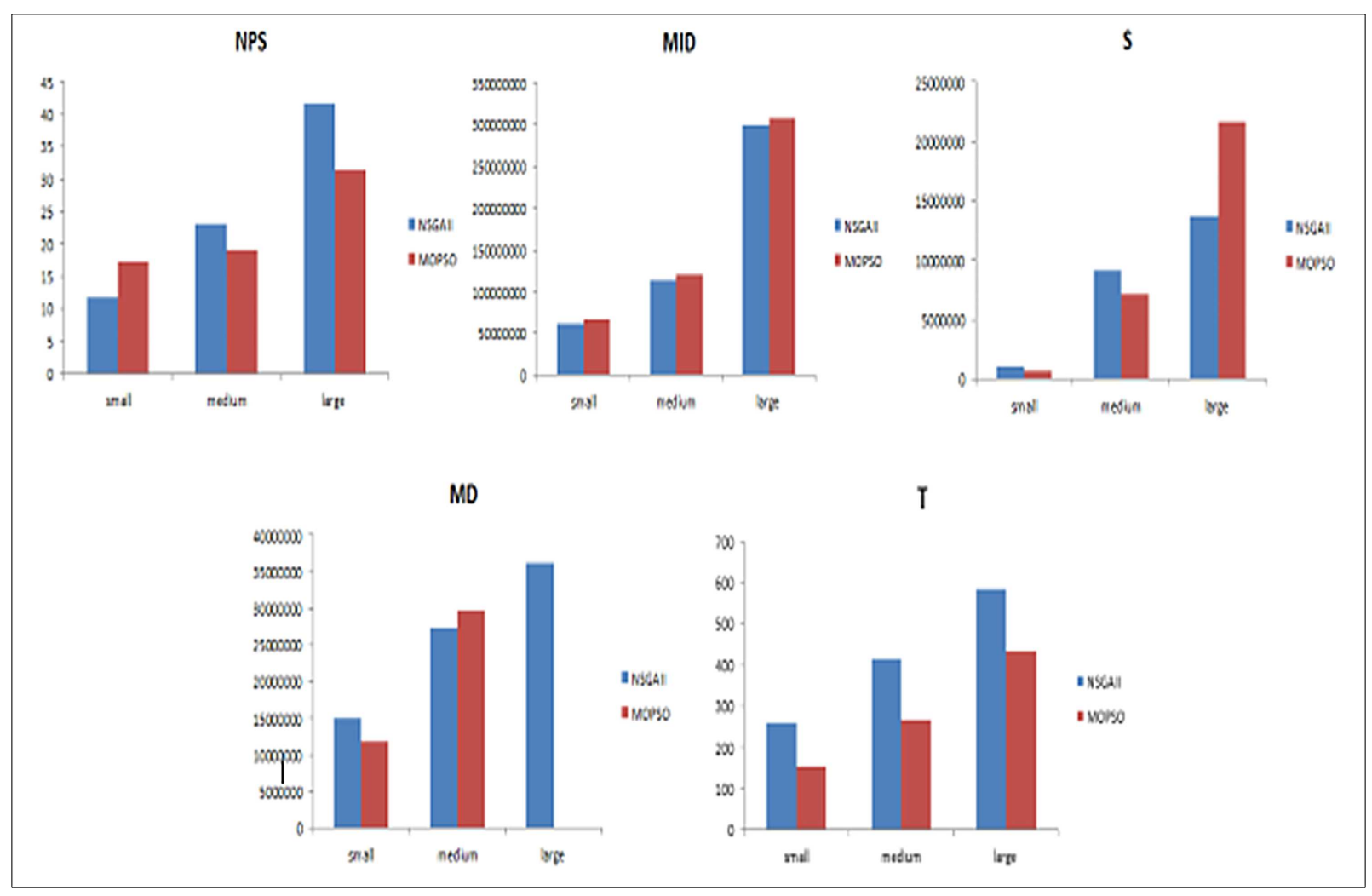

FiguRE 8. Average values of the 6 comparative criteria in different sizes.

- In NSGAII algorithm, the value of the NPS criterion is higher in medium and large sizes, which causes more alternative solutions to the supply chain managers and decision makers.

- The MID criterion in NSGAII algorithm is less than MOPSO algorithm in all sizes (with a small difference). Therefore, the NSGAII algorithm is more favorable in terms of MID. 
TABLE 24. Ranking of the algorithms in different sizes based on results of TOPSIS method.

\begin{tabular}{llllll}
\hline \hline Size & Algorithm & $d^{+}$ & $d^{-}$ & CL & Ranking \\
\hline \multirow{2}{*}{ Small } & NSGAII & $0 / 52$ & $0 / 18$ & $0 / 26$ & 2 \\
& MOPSO & $0 / 18$ & $0 / 52$ & $0 / 74$ & 1 \\
\hline \multirow{2}{*}{ Medium } & NSGAII & $0 / 34$ & $0 / 14$ & $0 / 3$ & 2 \\
& MOPSO & $0 / 14$ & $0 / 34$ & $0 / 7$ & 1 \\
\hline \multirow{2}{*}{ Large } & NSGAII & $0 / 21$ & $0 / 36$ & $0 / 63$ & 1 \\
& MOPSO & $0 / 36$ & $0 / 21$ & $0 / 37$ & 2 \\
\hline
\end{tabular}

- For small and medium problems, MOPSO algorithm has less value than solutions of NSGAII algorithm and for large problem, it is the vice versa.

- The fourth criterion is MD and the results show that for medium and large sizes, the MOPSO algorithm is more efficient.

- Since the MOPOS algorithm has a lower T value in all three sizes, it is superior to the NSGAII algorithm.

To compare the proposed algorithms and choose the best one in any size, TOPSIS method is utilized assuming that the weight of all comparative criteria is the same. Table 24 represents the obtained results.

As can be seen in Table 22, the NGAII algorithm has higher efficiency for small size problems and the MOPSO algorithm is more suitable for medium and large sizes.

\section{Conclusion}

This paper presents a new multi-objective model for designing a dynamic and three level blood supply chain network that aims to attain high quality of blood delivery at low time and cost. The main contribution of the proposed model is incorporating TQM principles clustered in four critical elements of the blood supply chain (costumer, process, equipment and staff) emphasizing on preventive maintenance, inspection and blood delivery quality and complexity of the supply chain inclusive various types of blood products with different lifetime derived from both whole blood and apheresis. Moreover, blood demand is modeled by belief structure of IER approach to handle the uncertainty. For computing solution and illustrating applicability of the model, the NSGAII and MOPSO algorithms are implemented on 15 numerical examples in different sizes. the obtained solutions are compared through TOPSIS method based on criteria inclusive NPS, MID, S, MD and T. Based on the results, it can be concluded that the NSGAII algorithm is more efficient for large size problem and for small and medium sizes; the MOPSO algorithm has a higher utility. For future studies, intricacies like compatibility of blood groups and horizontal collaboration can be addressed. In addition, the proposed model has potential for applications other metaheuristic algorithms and can be successfully implement in real case.

\section{Appendix A.}

In this section, the first objective function that aims to minimize the total cost is presented as follows:

$$
\begin{aligned}
\operatorname{Min} Z_{1}= & \sum_{j} \sum_{p} N_{j p}^{\prime} \times C P A_{j p}^{\prime} \times n e_{j p}^{\prime} \times Y_{j}^{\prime}+\sum_{i} F_{i} \times Y_{i}+\sum_{j} F_{j} \times Y_{j}^{\prime}+\sum_{i} \sum_{j} \sum_{t} \sum_{v}\left(L O_{i j}+L C_{i j}\right) Y_{i j t v} \\
& +\sum_{j} \sum_{k} \sum_{t} \sum_{v^{\prime}}\left(L O_{j k}^{\prime}+L C_{j k}^{\prime}\right) Y_{j k t v^{\prime}}^{\prime}+\sum_{i} \sum_{j} \sum_{t} \sum_{V} C T_{i j}^{v} \times d_{i j} \times Q_{i j t}+\sum_{j} \sum_{k} \sum_{t} \sum_{v^{\prime}} \sum_{m} \sum_{r_{m}} \\
& \times C T_{j k m}^{v^{\prime}} \times d_{j k}^{\prime} \times\left(Q_{j k t v^{\prime}}^{m, r_{m}}+Q A_{j k t v^{\prime}}^{m, r_{m}}\right)+\sum_{j} \sum_{t} \sum_{m=2} \sum_{r_{m}} C M_{j m} \times Q_{j, t, m, r_{m}}^{\prime}+\sum_{j} \sum_{t} \sum_{m=2} C M A_{j m} \\
& \times Q A_{j t m} \times \sum_{i} \sum_{t} C P_{i} \times Q_{i t} \times Y_{i}+\sum_{i} \sum_{j} \sum_{t} \sum_{v} C P_{j}^{\prime} \times\left(1-\alpha_{j t}^{(2)}\right) \times\left(1-\alpha_{i j t v}^{(1)}\right) \times Q_{i j t v} \times Y_{j}^{\prime}
\end{aligned}
$$




$$
\begin{aligned}
& +\sum_{j} \sum_{k} \sum_{t} \sum_{v^{\prime}} \sum_{m=2} \sum_{r_{m}} C P_{k}^{\prime \prime} \\
& \times\left[\left[\frac{\left(\sum_{j} \sum_{v^{\prime}} \sum_{m} \sum_{r_{m}}\left(1-\alpha_{j k t v^{\prime}}^{(1)}{ }^{m, r_{m}}\right) Q_{j k t v^{\prime}}^{m, r_{m}}+I n_{k(t-1)}^{m, r_{m}}\right) \times\left(1-\alpha_{k, t, m, r_{m}}^{(5)}\right) \times P C_{k, t, m, r_{m}}}{a}\right]\right. \\
& \left.\times a+\left(\left(1-\alpha_{j k t v^{\prime}}^{(2)}{ }^{m, r_{m}}\right) Q A_{j k t v^{\prime}}^{m, r_{m}}+I n a_{k(t-1)}^{m, r_{m}}\right) \times\left(1-\alpha_{k, t, m, r_{m}}^{(6)}\right) P C A_{k, t, m, r_{m}}\right] \\
& +\sum_{j} \sum_{k} \sum_{t} \sum_{v^{\prime}} \sum_{r_{1}} C P_{k}^{\prime \prime} \times\left(1-\alpha_{j k t v^{\prime}}^{(1)}{ }^{1, r_{1}} Q_{j k t v^{\prime}}^{1, r_{1}}+I n_{k(t-1)}^{1, r_{1}}\right) \times\left(1-\alpha_{k, t, 1, r_{1}}^{(5)}\right) \times P C_{k, t, 1, r_{1}}+\sum_{j} \sum_{t} C H_{j 1} \\
& \times I_{j t}+\sum_{j} \sum_{t} \sum_{m} \sum_{r_{m}} C H_{j m}\left(I S_{j t}^{m, r_{m}}\right)+\sum_{j} \sum_{t} \sum_{m=2} \sum_{r_{m}} C H_{j m} \times I A_{j t}^{m, r_{m}}+\sum_{k} \sum_{t} \sum_{m} \sum_{r_{m}} C H_{k m}^{\prime} \\
& \times\left(I n_{k t}^{m, r_{m}}+I n a_{k t}^{m, r_{m}}\right)+\sum_{k} \sum_{t} \sum_{m} \sum_{r_{m}} C S_{k m} \times Q \pi_{k t}^{m, r_{m}}+\sum_{k} \sum_{t} \sum_{m} \sum_{r_{m}} C S A_{k m} \times Q \pi a_{k t}^{m, r_{m}} \\
& +\sum_{j} \sum_{k} \sum_{t} \sum_{v^{\prime}} \sum_{m} \sum_{r_{m}} C L_{k}^{m, r_{m}} \times Y L_{j k t v^{\prime}} \times\left(Q_{j k t v^{\prime}}^{m, r_{m}}+Q A_{j k t v^{\prime}}^{m, r_{m}}\right)+\sum_{j} \sum_{k} \sum_{t} \sum_{v^{\prime}} \sum_{m} \sum_{r_{m}} C E_{k}^{m, r_{m}} \\
& \times Y E_{j k t v^{\prime}} \times\left(Q_{j k t v^{\prime}}^{m, r_{m}}+Q A_{j k t v^{\prime}}^{m, r_{m}}\right)+\sum_{i}\left(C P r_{i}+C E V_{i}+C E R_{i}\right) \times T \times Y_{i}+\sum_{j}\left(C P r_{j}^{\prime}+C E V_{j}^{\prime}\right. \\
& \left.+C E R_{j}^{\prime}+C E R P_{j}\right) \times T \times Y_{j}^{\prime}+\sum_{k}\left(C P r_{k}^{\prime \prime}+C E V_{k}^{\prime \prime}+C E R_{k}^{\prime \prime}\right) \times T+\sum_{i} \sum_{t} C W_{i} \times \alpha_{i t}^{(1)} \times Q_{i t} \\
& +\sum_{i} \sum_{j} \sum_{t} \sum_{v} C W_{j}^{\prime} \times \alpha_{j t}^{(2)} \times\left(1-\alpha_{i j t v}^{(1)}\right) \times Q_{i j t v}+\sum_{j} \sum_{t} \sum_{m=2} \sum_{r_{m}} C W_{j}^{m, r_{m}} \times\left[\alpha_{j, t, m, r_{m}}^{(2)} \times P Q_{j t m}\right. \\
& \left.+\left(1-P Q_{j t m}\right) \frac{\left(1-E P_{j}^{(1)}\right) \times \sum_{m} \sum_{r_{m}}\left[P_{j t m} \times\left(1-\alpha_{j m}^{(4)}\right) \times Q_{j, t, m, r_{m}}^{\prime}\right]}{\sum_{m} \sum_{r_{m}} Q_{j, t, m, r_{m}}^{\prime}}\right] \times P_{j t m} \times\left(1-\alpha_{j m}^{(4)}\right) \\
& \times Q_{j, t, m, r_{m}}^{\prime}+\sum_{i} \sum_{j} \sum_{t} \sum_{v} \sum_{r_{1}} C W_{j}^{1, r_{1}} \times \alpha_{j, t, 1, r_{1}}^{(2)} \times P Q_{j t 1}+\left(1-P Q_{j t 1}\right) \times \sum_{j} \sum_{t} \\
& \times\left[\frac{\left(1-E P_{j}^{(2)}\right) \times \sum_{i} \sum_{v} P_{j t 1} \times\left(1-\alpha_{j}^{(1)}-\alpha_{j}^{(2)}\right) \times \alpha_{j t}^{(3)} \times\left(1-\alpha_{j t}^{(2)}\right) \times\left(1-\alpha^{(1)}{ }_{i j t v}\right) \times Q_{i j t v}}{\sum_{i} \sum_{v} \alpha_{j t}^{(3)} \times\left(1-\alpha_{j t}^{(2)}\right) \times\left(1-\alpha_{i j t v}^{(1)}\right) \times Q_{i j t v}}\right] \\
& \times P_{j t 1} \times\left(1-\alpha_{j}^{(1)}-\alpha_{j}^{(2)}\right) \times \alpha_{j t}^{(3)} \times\left(1-\alpha_{j t}^{(2)}\right) \times\left(1-\alpha_{i j t v}^{(1)}\right) \times Q_{i j t v}+\sum_{j} \sum_{t} \sum_{m=2} \sum_{r_{m}} C W A_{j}^{m, r_{m}} \\
& \times P A_{j t m} \times\left(1-\alpha_{j m}^{(5)}\right) Q A_{j t m} \times\left[\alpha_{j t m} \times P Q A_{j t m}+\left(1-P Q A_{j t m}\right) \sum_{j} \sum_{t}\right. \\
& \left.\times \frac{\left(1-E P_{j}^{(3)}\right) \times \sum_{m} P A_{j t m} \times\left(1-\alpha_{j m}^{(5)}\right) Q A_{j t m}}{\sum_{m} Q A_{j t m}}\right]+\sum_{j} \sum_{t} \sum_{m=2} \sum_{r_{m}} C W_{j}^{m, r_{m}} \times\left[\left(1-\alpha_{j, t, m, r_{m}}^{(2)}\right)\right. \\
& \left.\times P Q_{j t m} \times P_{j t m} \times\left(1-\alpha_{j m}^{(4)}\right) \times Q_{j, t, m, r_{m}}^{\prime}+I S_{j(t-1)}^{m, r_{m}}\right] \times \alpha_{j, t, m, r_{m}}^{(3)}+\sum_{i} \sum_{j} \sum_{t} \sum_{v} \sum_{r_{1}} C W_{j}^{1, r_{1}} \\
& \times\left[\left(1-\alpha_{j, t, 1, r_{1}}^{(2)}\right) \times P Q_{j t 1} \times P_{j t 1} \times\left(1-\alpha_{j}^{(1)}-\alpha_{j}^{(2)}\right) \times \alpha_{j t}^{(3)} \times\left(1-\alpha_{j t}^{(2)}\right) \times\left(1-\alpha_{j t}^{(1)}\right) \times Q_{i j t v}+I S_{j(t-1)}^{1, r_{1}}\right] \\
& \times \alpha_{j, t, 1, r_{1}}^{(3)}+\sum_{j}+\sum_{j}+\sum_{m=2} \sum_{r_{m}} C W A_{j}^{m, r_{m}} \\
& \times\left[P A_{j t m} \times\left(1-\alpha_{j m}^{(5)}\right) Q A_{j t m} \times\left(1-\alpha_{j t m}\right) \times P Q A_{j t m}+I A_{j(t-1)}^{m, r_{m}}\right] \times \alpha_{j, t, m, r_{m}}^{(4)}
\end{aligned}
$$




$$
\begin{aligned}
& +\sum_{j} \sum_{k} \sum_{t} \sum_{v^{\prime}} \sum_{m} \sum_{r_{m}} C W_{k}^{\prime m, r_{m}} \times\left[\left(1-\alpha_{j k t v^{\prime}}^{(1)}{ }^{m, r_{m}}\right)\left(Q_{j k t v^{\prime}}^{m, r_{m}}\right)+I n_{k(t-1)}^{m, r_{m}}\right] \times \alpha_{k, t, m, r_{m}}^{(5)} \\
& +\sum_{j} \sum_{k} \sum_{t} \sum_{v^{\prime}} \sum_{m} \sum_{r_{m}} C W_{k}^{\prime m, r_{m}} \times\left[\left(1-\alpha_{j k t v^{\prime}}^{(1)}{ }^{m, r_{m}}\right)\left(Q_{j k t v^{\prime}}^{m, r_{m}}\right)+I n_{k(t-1)}^{m, r_{m}}\right] \times \alpha_{k, t, m, r_{m}}^{(5)}
\end{aligned}
$$

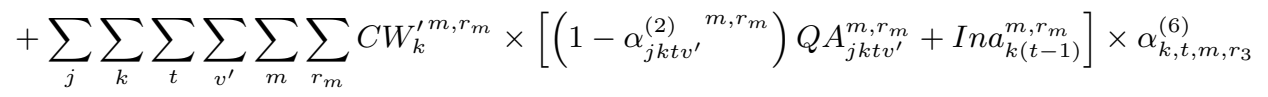

$$
\begin{aligned}
& +\sum_{j} \sum_{k} \sum_{t} \sum_{v^{\prime}} \sum_{r_{1}} C W_{k}^{\prime 1, r_{1}}\left(\left(1-\alpha_{j k t v^{\prime}}^{(1)}{ }^{1, r_{1}}\right) Q_{j k t v^{\prime}}^{1, r_{1}}+I n_{k(t-1)}^{1, r_{1}}\right) \times\left(1-\alpha_{k, t, 1, r_{1}}^{(5)}\right) \times P C_{k, t, 1, r_{1}} \\
& -Q T_{k, t, r_{1}}+\sum_{j} \sum_{k} \sum_{t} \sum_{v^{\prime}} \sum_{m} \sum_{r_{m}} C R E P_{j k}^{m, r_{m}} \times\left[\alpha_{j k t v^{\prime}}^{(1) r^{m}}\left(Q_{j k t v^{1}}^{m, r_{m}}\right)+\alpha_{j k t v^{\prime}}^{(2)}{ }^{m, r_{m}}\left(Q A_{j k t v^{\prime}}^{m, r_{m}}\right)\right] \\
& +\sum_{i} \sum_{j} \sum_{t} \sum_{v} C R E P_{i j} \times \alpha_{i j t v}^{(1)} \times Q_{i j t v} \times \sum_{j} \sum_{t} \sum_{m=2} \sum_{r_{m}} C M I_{j m} \times\left[\left(1-\alpha_{j m}^{(4)}\right) \times Q_{j, t, m, r_{m}}^{\prime}+\left(1-\alpha_{j m}^{(5)}\right)\right. \\
& \left.\times Q A_{j t m}\right]+\sum_{j} \sum_{t} \sum_{m=2} \sum_{r_{m}} C M I_{j m} \times\left[\left(1-\alpha_{j m}^{(4)}\right) \times Q_{j, t, m, r_{m}}^{\prime}+\left(1-\alpha_{j m}^{(5)}\right) Q A_{j t m}\right]+\sum_{i} \sum_{j} \sum_{t} \sum_{v} C M I_{j 1} \\
& \times\left(1-\alpha_{j}^{(1)}-\alpha_{j}^{(2)}\right) \times \alpha_{j t}^{(3)} \times\left(1-\alpha_{j t}^{(2)}\right) \times\left(1-\alpha_{i j t v}^{(1)}\right) \times Q_{i j t v}+\sum_{i} \sum_{o} N_{i o} \times C P A_{i o} \times n e_{i o} \\
& \times Y_{i} \sum_{k} \sum_{q} N_{k q}^{\prime \prime} \times C P A_{k q}^{\prime \prime} \times n e_{k q}^{\prime \prime}+\sum_{i} \sum_{o} C B_{o} \times\left[\left(1-P D R_{i o}\right)+P D R_{i o} \times\left(1-P R_{i o}\right)\right] \times \lambda_{i o} \times T \\
& \times Y_{i}+\sum_{j} \sum_{p} C B_{P}^{\prime} \times\left[\left(1-P D R_{j p}^{\prime}\right)+P D R_{j p}^{\prime} \times\left(1-P R_{j p}^{\prime}\right)\right] \times \lambda_{j p}^{\prime} \times T \times Y_{j}^{\prime}+\sum_{k} \sum_{q} C B_{q}^{\prime \prime} \\
& \times\left[\left(1-P D R_{k q}^{\prime \prime}\right)+P D R_{k q}^{\prime \prime} \times\left(1-P R_{k q}^{\prime \prime}\right)\right] \times \lambda_{k y}^{\prime \prime} \times T+\sum_{i} \sum_{o} \sum_{t} C R_{i o} \times \lambda_{i o} \times T \times P D R_{i o} \times Y_{i} \\
& +\sum_{j} \sum_{p} \sum_{t} C R_{j p}^{\prime} \times \lambda_{j p}^{\prime} \times T \times P D R_{j p}^{\prime} \times Y_{j}^{\prime}+\sum_{k} \sum_{q} C R_{k q}^{\prime \prime} \times \lambda_{k q}^{\prime \prime} \times T \times P D R_{k q}^{\prime \prime}+\sum_{j} \sum_{t} \sum_{m=2} \sum_{r_{m}} \\
& \times C Q C_{j m} \times\left(\alpha_{j m}^{(4)} \times Q_{j, t, m, r_{m}}^{\prime}+\alpha_{j m}^{(5)} \times Q A_{j t m}\right)+\sum_{i} \sum_{j} \sum_{t} \sum_{v} C Q C_{j 1} \times \alpha_{j}^{(1)} \times \alpha_{j t}^{(3)} \times\left(1-\alpha_{j t}^{(2)}\right) \\
& \times\left(1-\alpha_{i j t v}^{(1)}\right) \times Q_{i j t v}+\sum_{j} \sum_{t} \sum_{m=2} \sum_{r_{m}} C A_{j m}\left[P Q_{j m} \times\left(1-\alpha_{j, t, m, r_{m}}^{(2)}\right) \times P_{j m} \times\left(1-\alpha_{j m}^{(4)}\right)\right. \\
& \left.\times Q_{j, t, m, r_{m}}^{\prime}\right] \times \frac{E p_{j}^{(1)} \times \sum_{m} \sum_{r_{m}}\left[P Q_{j m} \times\left(1-\alpha_{j, t, m, r_{m}}^{(2)}\right) \times P_{j m} \times\left(1-\alpha_{j m}^{(4)}\right) \times Q_{j, t, m, r_{m}}^{\prime}\right]}{\sum_{m} \sum_{r_{m}} Q_{j, t, m, r_{m}}^{\prime}} \\
& +\sum_{i} \sum_{j} \sum_{t} \sum_{v} \sum_{r_{1}} C A_{j 1} \times\left[P Q_{j 1} \times\left(1-\alpha_{j, t, 1, r_{1}}^{(2)}\right) \times P_{j 1} \times\left(1-\alpha_{j}^{(1)}-\alpha_{j}^{(2)}\right)\right. \\
& \left.\times \alpha_{j t}^{(3)} \times\left(1-\alpha_{j t}^{(2)}\right) \times\left(1-\alpha_{i j t v}^{(1)}\right) \times Q_{i j t v}\right] \\
& ++\times \frac{E p_{j}^{(2)} \times \sum_{i} \sum_{v} P Q_{j 1} \times\left(1-\alpha_{j, t, 1, r_{1}}^{(2)}\right) \times P_{j 1} \times\left(1-\alpha_{j}^{(1)}-\alpha_{j}^{(2)}\right) \times \alpha_{j t}^{(3)} \times\left(1-\alpha_{j t}^{(2)}\right) \times\left(1-\alpha_{i j t v}^{(1)}\right) \times Q_{i j t v}}{\sum_{i} \sum_{v} \alpha_{j t}^{(3)} \times\left(1-\alpha_{j t}^{(2)}\right) \times\left(1-\alpha_{i j t v}^{(1)}\right) \times Q_{i j t v}} \\
& +\sum_{j} \sum_{t} \sum_{m=2} C A A_{j t} \times\left[P Q_{j m} \times\left(1-\alpha_{j t m}\right) \times P A_{j m} \times\left(1-\alpha_{j m}^{(5)}\right) \times Q A_{j t m}\right] \\
& \times \frac{E P_{j}^{(3)} \times \sum_{m}\left[P Q_{j m} \times\left(1-\alpha_{j t m}\right) \times P A_{j m} \times\left(1-\alpha_{j m}^{(5)}\right) \times Q A_{j t m}\right]}{\sum_{m} Q A_{j t m}} .
\end{aligned}
$$




\section{REFERENCES}

[1] M.M. Aldaihani and M.A. Darwish, Effect of preventive maintenance on supply chains with one producer and multiple newsvendors. Int. J. Appl. Manage. Sci. 7 (2015) 19-37.

[2] E. Alfonso, X. Xie, V. Augusto and O. Garraud, Modeling and simulation of blood collection systems. Health Care Manage. Sci. 15 (2012) 63-78.

[3] P. Amorim, H-O. Günther and B. Almada-Lobo, Multi-objective integrated production and distribution planning of perishable products. Int. J. Prod. Econ. 138 (2012) 89-101.

[4] M. Arvan, R. Tavakkoli-Moghaddam and M. Abdollahi, Designing a bi-objective and multi-product supply chain network for the supply of blood. Uncertain Supply Chain Manage. 3 (2015) 57-68.

[5] M. Casadesús and R de Castro, How improving quality improves supply chain management: empirical study. TQM Mag. $\mathbf{1 7}$ (2005) 345-357.

[6] E. Cetin and L.S. Sarul, A blood bank location model: a multi-objective approach. Eur. J. Pure Appl. Math. 2 (2009) 112-124.

[7] J. Chen, W. Zhang and Z. Liu, Joint pricing, services and quality decisions in a dual-channel supply chain. RAIRO:OR $\mathbf{5 4}$ (2020) 1041-1056.

[8] K.S. Chin, Y.M. Wang, J.B. Yang and K.K.G. Poon, An evidential reasoning based approach for quality function deployment under uncertainty. Expert Syst. App. 36 (2009) 5684-5694.

[9] C.A.C. Coello, G.T. Pulido and M.S. Lechuga, Handling multiple objectives with particle swarm optimization. IEEE Trans. Evol. Comput. 8 (2004) 256-279.

[10] C. Da, Y. Cheng and C. Ying, An analysis on the inventory management of blood scheduling after Wen Chuan Earthquake. Chin. J. Blood Transfusion (2002) 92-22.

[11] M.S. Daskin, C.R. Coullard and Z.J.M. Shen, An inventory-location model: formulation, solution algorithm and computational results. Ann. Oper. Res. 110 (2002) 83-106.

[12] K. Deb, A. Pratap, S. Agarwal and T.A.M.T. Meyarivan, A fast and elitist multiobjective genetic algorithm: NSGA-II. IEEE Trans. Evol. Comput. 6 (2002) 182-197.

[13] A.P. Dempster, Upper and lower probabilities induced by a multivalued mapping. In: Classic Works of the Dempster-Shafer Theory of Belief Functions. Vol. 219 of Studies in Fuzziness and Soft Computing. Springer, Berlin-Heidelberg (2008) 57-72.

[14] P. Farahani, M. Grunow and H.-O. Günther, Integrated production and distribution planning for perishable food products. Flexible Serv. Manuf. J. 24 (2012) 28-51.

[15] B.B. Flynn and E.J. Flynn, Synergies between supply chain management and quality management: emerging implications. Int. J. Prod. Res. 43 (2005) 3421-3436.

[16] L.B. Forker, D. Mendez and J.C. Hershauer, Total quality management in the supply chain: what is its impact on performance. Int. J. Prod. Res. 35 (1997) 1681-1702.

[17] S.T. Foster Jr, Towards an understanding of supply chain quality management. J. Oper. Manage. 26 (2008) $461-467$.

[18] B. Fynes, C. Voss and S. de Búrca, The impact of supply chain relationship quality on quality performance. Int. J. Prod. Econ. 96 (2005) 339-354.

[19] S. Gunpinar and G. Centeno, Stochastic integer programming models for reducing wastages and shortages of blood products at hospitals. Comput. Oper. Res. 54 (2015) 129-141.

[20] V.C. Hemmelmayr, K.F. Doerner and R.F. Hartl, A variable neighborhood search heuristic for periodic routing problems. Eur. J. Oper. Res. 195 (2009) 791-802.

[21] V.R. Kannan and K.C. Tan, Just in time, total quality management, and supply chain management: understanding their linkages and impact on business performance. Omega 33 (2005) 153-162.

[22] A. Kaveh and M. Ghobadi, A multistage algorithm for blood banking supply chain allocation problem. Int. J. Civil Eng. 15 (2017) 103-112.

[23] H. Kaynak and J.L. Hartley, A replication and extension of quality management into the supply chain. J. Oper. Manage. 26 (2008) 468-489.

[24] K.E. Kendall and S.M. Lee, Formulating blood rotation policies with multiple objectives. Manage. Sci. 26 (1980) $1145-1157$.

[25] A. Khosrojerdi, S.H. Zegordi, J.K. Allen and F. Mistree, A method for designing power supply chain networks accounting for failure scenarios and preventive maintenance. Eng. Optim. 48 (2016) 154-172.

[26] C. Lin, W.S. Chow, C.N. Madu, C.H. Kuei and P.P.A. Yu, Structural equation model of supply chain quality management and organizational performance. Int. J. Prod. Econ. 96 (2005) 355-365.

[27] V.H. Lo and A. Yeung, Managing quality effectively in supply chain: a preliminary study. Supply Chain Manage. Int. J. 11 (2006) 208-215.

[28] S.N.A.A. Malik, H. Musa, S. Ahmad and N. Mohamad, The factors influencing supply chain disruptions on supply chain performance in small and medium enterprises. J. Technol. Manage. Technopreneurship 2 (2014).

[29] R.M. Monczka, R.B. Handfield, L.C. Giunipero and J.L. Patterson, Purchasing and supply chain management. In: Purchasing and Supply Chain Management, 4th edition. South-Western Cengage Learning, Mason, OH (2009).

[30] A.F.O. Muriel, S. Brailsford and H. Smith, A bi-objective optimization model for technology selection and donor's assignment in the blood supply chain. Sistemas Telemática 12 (2014) 9-24.

[31] A. Nagurney and A.H. Masoumi, Supply chain network design of a sustainable blood banking system. In: Sustainable Supply Chains. Vol.174 of International Series in Operations Research \& Management Science. Springer, New York, NY (2012) 49-72. 
[32] A. Nagurney, A.H. Masoumi and M. Yu, Supply chain network operations management of a blood banking system with cost and risk minimization. Comput. Manage. Sci. 9 (2012) 205-231.

[33] M. Ogier, V.D. Cung and J. Boissiere, Service network design in short and local fresh food supply chain. RAIRO:OR 47 (2019) 445-464.

[34] W.P. Pierskalla, Supply chain management of blood banks. In: Operations Research and Health Care. Vol.70 of International Series in Operations Research \& Management Science. Springer, Boston, MA (2005) 103-145.

[35] M.J. Pires, P. Amorim, S. Martins and B. Almada-Lobo, Production planning of perishable food products by mixed-integer programming. In: Operational Research. Vol. 4 of CIM Series in Mathematical Sciences. Springer, Cham (2015) 331-352.

[36] P. Pourkarim Guilani, Using NSGA II algorithm for a three objectives redundancy allocation problem with k-out-of-n subsystems. J. Optim. Ind. Eng. 9 (2016) 87-96.

[37] C.J. Robinson and M.K. Malhotra, Defining the concept of supply chain quality management and its relevance to academic and industrial practice. Int. J. Prod. Econ. 96 (2005) 315-337.

[38] P. Romano and A. Vinelli, Quality management in a supply chain perspective: strategic and operative choices in a textileapparel network. Int. J. Oper. Prod. Manage. 21 (2001) 446-460.

[39] G. Şahin, H. Süral and S. Meral, Locational analysis for regionalization of Turkish Red Crescent blood services. Comput. Oper. Res. 34 (2007) 692-704.

[40] F.G. Şahinyazan, B.Y. Kara and M. Taner, Selective vehicle routing for a mobile blood donation system. Eur. J. Oper. Res. 245 (2015) 22-34.

[41] A. Salehipour and M.M. Sepehri, Exact and heuristic solutions to minimize total waiting time in the blood products distribution problem. Adv. Oper. Res. 2012 (2012) 393890.

[42] G. Shafer, A Mathematical Theory of Evidence. Princeton University Press, Princeton, NJ (1976).

[43] Z.J.M. Shen, C. Coullard and M.S. Daskin, A joint location-inventory model. Transp. Sci. 37 (2003) 40-55.

[44] D.L. Xu, J.B. Yang and Y.M. Wang, The evidential reasoning approach for multi-attribute decision analysis under interval uncertainty. Eur. J. Oper. Res. 174 (2006) 1914-1943.

[45] J.B. Yang and M.G. Singh, An evidential reasoning approach for multiple-attribute decision making with uncertainty. IEEE Trans. Syst. Man Cybern. 24 (1994) 1-18.

[46] J.B. Yang, Y.M. Wang, D.L. Xu and K.S. Chin, The evidential reasoning approach for MADA under both probabilistic and fuzzy uncertainties. Eur. J. Oper. Res. 171 (2006) 309-343.

[47] B. Zahiri and M.S. Pishvaee, Blood supply chain network design considering blood group compatibility under uncertainty. Int. J. Prod. Res. 55 (2017) 2013-2033.

[48] A. Zeinalzadeh, Y. Mohammadi and M.H. Moradi, Optimal multi objective placement and sizing of multiple DGs and shunt capacitor banks simultaneously considering load uncertainty via MOPSO approach. Int. J. Electr. Power Energy Syst. 67 (2015) 336-349. 